\title{
Senescent macrophages in the human adipose tissue as a source of inflammaging
}

\author{
Giulia Matacchione ${ }^{\circledR} \cdot$ Jessica Perugini $~ E$ Eleonora Di Mercurio · Jacopo Sabbatinelli • \\ Francesco Prattichizzo • Martina Senzacqua - Gianluca Storci - Christian Dani • Giovanni Lezoche • \\ Mario Guerrieri • Antonio Giordano $\cdot$ Massimiliano Bonafè $\cdot$ Fabiola Olivieri
}

Received: 14 September 2021 / Accepted: 21 February 2022 / Published online: 5 March 2022

(C) The Author(s) 2022, corrected publication 2022

\begin{abstract}
Obesity is a major risk factor for type 2 diabetes and a trigger of chronic and systemic inflammation. Recent evidence suggests that an increased burden of senescent cells (SCs) in the adipose tissue of obese/diabetic animal models might underlie such pro-inflammatory phenotype. However, the role of macrophages as candidate SCs, their phenotype, the distribution of SCs among fat depots, and clinical relevance are debated. The senescence marker
\end{abstract}

Giulia Matacchione, Jessica Perugini, Massimiliano Bonafè, and Fabiola Olivieri equally contributed to the manuscript.

Supplementary Information The online version contains supplementary material available at https://doi. org/10.1007/s11357-022-00536-0.

G. Matacchione $(\bowtie) \cdot$ J. Sabbatinelli $\cdot$ F. Olivieri Department of Clinical and Molecular Sciences, DISCLIMO, Università Politecnica delle Marche, Via Tronto 10/A, Ancona, Italy

e-mail: g.matacchione@pm.univpm.it

J. Perugini - E. Di Mercurio · M. Senzacqua - A. Giordano Department of Experimental and Clinical Medicine, Center of Obesity, Università Politecnica delle Marche, Ancona, Italy

F. Prattichizzo

IRCCS MultiMedica, Milano, Italy

G. Storci

IRCCS Azienda Ospedaliero-Universitaria di Bologna,

Bologna, Italy $\beta$-galactosidase and the macrophage marker CD68 were scored in visceral (vWAT) and subcutaneous (scWAT) adipose tissue from obese patients $(n=17)$ undergoing bariatric surgery and control patients $(n=4)$ subjected to cholecystectomy. A correlation was made between the number of SCs and BMI, serum insulin, and the insulin resistance (IR) index HOMA. The monocyte cell line (THP-1) was cultured in vitro in high glucose milieu (60 mM D-glucose) and subsequently co-cultured with human adipocytes (hMADS) to investigate the reciprocal inflammatory activation. In obese patients, a significantly higher number of SCs was observed in vWAT compared to scWAT; about $70 \%$ of these cells expressed the macrophage marker CD68; and the number of SCs in vWAT, but not in scWAT, positively correlated with

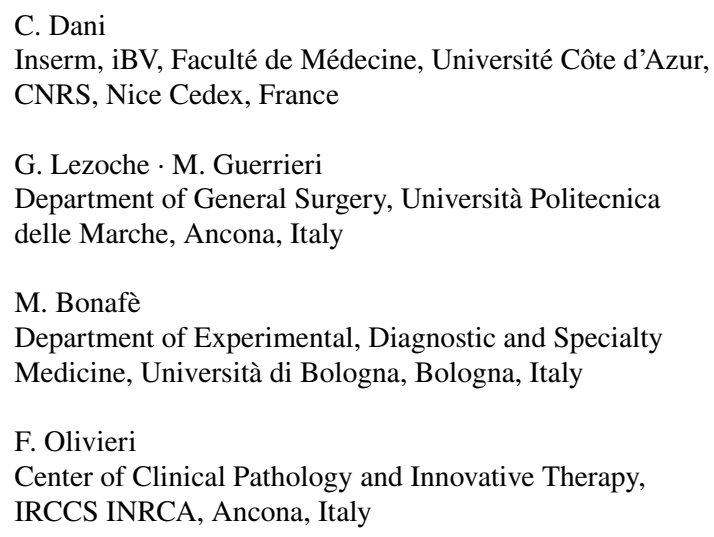


BMI, HOMA-IR, and insulin. THP-1 cultured in vitro in high glucose milieu acquired a senescent-like phenotype (HgSMs), characterized by a polarization toward a mixed M1/M2-like secretory phenotype. Coculturing $\mathrm{HgSMs}$ with hMADS elicited pro-inflammatory cytokine expression in both cell types, and defective insulin signaling in hMADS. In morbid obesity, expansion of visceral adipose depots involves an increased burden of macrophages with senescent-like phenotype that may promote a pro-inflammatory profile and impair insulin signaling in adipocytes, supporting a framework where senescent macrophages fuel obesity-induced systemic inflammation and possibly contribute to the development of IR.

Keywords Senescent cell $\cdot$ Macrophage - Obesity · Insulin resistance $\cdot$ Inflammaging $\cdot$ Adipose tissue

\section{Introduction}

Inflammaging is a portmanteau word that was minted to depict the chronic, sterile, low-grade inflammatory status that characterizes the human aging process $[1,2]$. A state of systemic hyper-inflammation has been also reported in a large number of age-related dysfunctions and diseases (ARDs), such as in obese subjects and in people affected by type 2 diabetes mellitus, T2DM [3, 4]. In turn, overnutrition/obesity and chronological aging can synergize in accelerating the rate of inflammaging, thus suggesting that inflammatory mechanisms at the basis of insulin resistance (IR)/T2DM, obesity, and aging are very difficult to disentangle $[5,6]$. In the recent years, age-related accrual of senescent cell (SC) burden in vivo gained the spotlight, as it was demonstrated that their selective ablation in mice is capable to delay aging features in tissue and organs [7]. In vitro, the loss of replicative capacity in SCs is accompanied by the onset of a pro-inflammatory senescence-associated secretory phenotype (SASP) [8]. In vivo, SASP is indicted to contribute to inflammaging and to play a crucial role in the onset and prognosis of diabetic complications [9-11].

Among SASP factors, a small number of miRNA, named inflamma-miRs and comprising miR-21, and miR-146a, are involved in regulating both cellular senescence and organismal inflammaging [12, 13]. During aging, inflamma-miR expression was found to be upregulated to restrain the tissue damage induced by the low-level chronic inflammation. The abnormal level of miR-21 and miR-146a has been observed both in normal aging and in the major ARDs [14, 15]. In both mice model and observational studies in humans, miR21 and miR-146a expression levels were consistently associated with both obesity and T2DM [16-19].

White adipose tissue (WAT) cells are highly susceptible to undergo senescence not only with aging but also with obesity and T2DM, independently of chronological age [20]. WAT senescence is associated with dysfunctional expansion (hypertrophy) of adipocytes, IR, and dyslipidemia. Recently, SCs have been found to accumulate in the adipose tissue of obese and diabetic human and mice [21, 22].

In the adipose tissue, features of senescence, such as a higher level of senescence-associated $\beta$-galactosidase (SA- $\beta-$ Gal) activity, and p53 and p21 protein levels have been documented $[23,24]$. Of note, the increase in the SC burden observed in diabetic mice was associated with impaired glucose tolerance, accrual of macrophages in the adipose tissue, and elevated production of pro-inflammatory mediators, while SC removal or attenuation of their phenotype improved metabolic homeostasis $[11,25]$. However, few studies have explored the burden of SCs in human fat samples and their relevance in relation to IR [9]. In addition, the cell type responsible for the SC burden observed in fat tissue is still debated, being pre-adipocytes, fully differentiated adipocytes, endothelial cells, macrophages, or other infiltrating immune cells possible candidates [26]. To address these issues, we aimed at identifying and quantifying the burden of senescent macrophages in visceral and subcutaneous adipose tissue samples obtained from obese and control patients, exploring their correlation with IR. Then, we exposed the human monocytic cell line (THP-1) to high glucose, an over-nutrition mimicking stimulus [10], to characterize the resulting phenotype and to set up a co-culture with human multipotent adipose-derived stem cells (hMADS), in order to evaluate the reciprocal influence on inflammation and insulin sensitivity.

\section{Materials and methods}

\section{Patients}

For histological analysis, adipose tissue (AT) biopsies were obtained from 17 severely obese patients (BMI $\geq$ 
$35 \mathrm{~kg} / \mathrm{m}^{2}$, i.e., obesity class II) and 4 control subjects $\left(\right.$ BMI $\leq 30 \mathrm{~kg} / \mathrm{m}^{2}$ ) undergoing respectively bariatric and non-bariatric (i.e., cholecystectomy) surgery at the General Hospital Azienda Ospedaliera Universitaria (AOU Ospedali Riuniti; Ancona, Italy). Samples from visceral (vWAT, omental depot) and subcutaneous (scWAT, abdominal region) adipose tissue were collected. The study protocol was approved by the Ethics Committee of Ospedali Riuniti (Ancona, Italy). Obesity was diagnosed and classified according to the BMI criteria. Subjects on anti-inflammatory drugs were excluded from the study. The information collected included anthropometric data and medical history (Table 1).

SA- $\beta$-galactosidase staining for fresh tissue and morphometry

AT biopsies (3-5-mm thickness) were fixed for $1 \mathrm{~h}$ with a fixative solution provided by the Senescence detection kit (Biovision Inc., Milpitas, CA, USA). After washing with PBS, samples were stained with the staining solution (X-Gal substrate: $20 \mathrm{mg} / \mathrm{ml}$ ), provided by the Senescence detection kit (Biovision), and incubated at $37^{\circ} \mathrm{C}$ (without $\mathrm{CO}_{2}$ ) for $20-22 \mathrm{~h}$. After, AT samples were first fixed in $4 \%$ paraformaldehyde overnight at $4{ }^{\circ} \mathrm{C}$ and then embedded in paraffin blocks.

From each adipose specimen, serial paraffin sections ( $4 \mu \mathrm{m}$ thick) were obtained and counterstained with Fast Red (Sigma-Aldrich, Milano, Italy). Tissue sections were examined with a Nikon Eclipse E800 light microscope and digital images were captured

Table 1 Comparison of biochemical and anthropometric characteristics among normal weight and obese subjects

\begin{tabular}{lll}
\hline Groups & Normal weight & Obese \\
\hline Sex, M/F, N/N & $0 / 4$ & $6 / 11$ \\
Age, year & $76.3 \pm 4.6$ & $47.6 \pm 2.2$ \\
Body mass index, kg/m² & $27.8 \pm 2.3$ & $45.58 \pm 0.91$ \\
Total cholesterol, mg/ml & $180 \pm 5.2$ & $180.2 \pm 8.4$ \\
Triglycerides, mg/dl & $98.4 \pm 8.3$ & $121.9 \pm 9.9$ \\
HbA1c, \% & $5.7 \pm 0.4$ & $6.56 \pm 0.7$ \\
Fasting glucose, mg/ml & $93.7 \pm 6.3$ & $100.33 \pm 4.4$ \\
Fasting insulin, $\mathrm{mcUI} / \mathrm{ml}$ & $5.4 \pm 1.7$ & $35.5 \pm 2.7$ \\
HOMA index & $1.25 \pm 0.9$ & $8.2 \pm 5.1$ \\
\hline
\end{tabular}

$N$ is 4 for normal-weight group and 17 for obese group. Variables are expressed as mean \pm SEM with a Nikon camera (DXM 1200). Senescenceassociated (SA)- $\beta$-galactosidase (Gal) positive (+) cells and adipocytes were counted by optical microscopy. The number of senescent cells was expressed as percentage in relation to adipocytes by counting the number of SA- $\beta-\mathrm{Gal}+$ cells and the number of adipocytes in at least 20 fields. The analysis excluded cells within and around blood vessels.

\section{CD68 and $p 16^{I N K 4 a_{i m m u n o s t a i n i n g}}$}

For immunohistochemistry, fixed AT samples were embedded with paraffin. Paraffin sections, $4 \mu \mathrm{m}$ thick, were reacted with 3\% hydrogen peroxide in PBS for $10 \mathrm{~min}$, and antigen-retrieved using citrate buffer (ph 6.0) in a steamer for 45 min. After blocking with $3 \%$ horse normal serum, sections were incubated overnight at $4{ }^{\circ} \mathrm{C}$ with anti-CD68 (Dako, Glostrup, Denmark) primary antibody $(1: 200)$ and $16^{\text {INK4a }}$ (ThermoFisher Scientific, Massachusetts, USA) primary antibody (1:800). Bound primary antibody was detected using a biotinylated anti-mouse IgG secondary antibody, $\mathrm{ABC}$ reagent (Vector Laboratories, Burlingame, USA), and DAB Substrate kit (SigmaAldrich) according to the manufacturer's instructions. Finally, sections were counterstained with Fast Red and mounted. CD68 + cells were counted among SA- $\beta-\mathrm{Gal}+$ cells in the entire tissue section. The analysis excluded cells within and around blood vessels.

THP-1 culture, macrophage polarization, and high-glucose concentration exposure treatment

Human monocytic THP-1 cells were purchased from ATCC (Rockville, MD, USA) and maintained in RPMI-1640 medium supplemented with 2-mercaptoethanol to a final concentration of $0.05 \mathrm{mM}$ and with $10 \%$ heat-inactivated fetal bovine serum (FBS), $1 \times$ penicillin/streptomycin $(100 \times)$, and $1 \times$ L-glutamine (100×) (all from Euroclone, Milano, Italy).

To induce polarization of THP- 1 cells into the M0 phenotype macrophages, cells were treated with phorbol 12-myristate 13-acetate (PMA) (100 ng/ml) for $24 \mathrm{~h}$. Afterwards, LPS (15 ng/ml) in RPMI medium with $10 \%$ FBS was added for $48 \mathrm{~h}$ to polarize M0 macrophages into the M1 phenotype (pro-inflammatory), whereas the M2 phenotype (anti-inflammatory) 
was induced with IL-4 (25 ng/ml) and IL-13 (25 ng/ $\mathrm{ml}$ ) in RPMI medium with 10\% FBS for $48 \mathrm{~h}$. THP-1 cells were maintained in high-glucose medium (60 $\mathrm{mM}$ ) for 1 week and then treated with $100 \mathrm{ng} / \mathrm{ml}$ PMA for $24 \mathrm{~h}$, to mimic hyperglycemia and to obtain macrophages (HgSMs). In order to examine THP-1 proliferation after 1 week treatment with $60 \mathrm{mM}$ D-glucose, cells were plated in 12-well plates at a density of 200,000 cells $/ \mathrm{ml}$, treated without or with $60 \mathrm{mM}$ of D-glucose (NG and HG respectively) and then counted after 1 week treatment. The experiment was performed in triplicate.

\section{Cell viability assay}

MTT (3-(4,5-dimethylthiazol-2-yl)-2,5-diphenyltetrazolium bromide) assay was used to test cell viability and proliferation of THP- 1 cells treated with 60 $\mathrm{mM}$ D-glucose. Cells were grown in 96-well plates at a density of 10000 cells/well and treated with or without $60 \mathrm{mM}$ of D-glucose. After 1 week, MTT (1 $\mathrm{mg} / \mathrm{ml}$ ) solution was added and incubated for $4 \mathrm{~h}$; the insoluble formazan salt product was solubilized by adding $200 \mu \mathrm{l}$ of dimethyl sulfoxide (DMSO) and its amount was determined by measuring the optical density at $540 \mathrm{~nm}$ using a microplate reader (MPT Reader, Invitrogen, Milano, Italy).

Cell viability was calculated according to the equation $(T / C) \times 100 \%$, where $T$ and $C$ represent respectively the mean optical density of the treated group and the control group.

hMADS cell culture and adipocyte differentiation

hMADS cells were cultured and differentiated as previously described [27]. Briefly, hMADS cells were grown in low-glucose $(1 \mathrm{~g} / \mathrm{l})$ proliferation medium (DMEM) supplemented with $10 \%$ fetal bovine serum and $2.5 \mathrm{ng} / \mathrm{ml}$ hFGF-2. For differentiation, hMADS cells were used between the 16th and the 19th passage. To induce adipose differentiation, they were seeded in proliferation medium on 6-well cell culture plates at a density of $4500 \mathrm{cells} / \mathrm{cm}^{2}$. When they reached confluence, hFGF-2 was not replaced. The next day (day 0), cells were incubated in adipogenic medium and cell lipid content was assessed by Oil Red O staining as previously described [27].
hMADS adipocytes and THP-1 co-culture system

hMADS cells were grown to confluency in 6-well cell culture plates and differentiated to mature adipocytes as described above. Adipocytes were then co-cultured in a transwell system with THP-1 cells differentiated into M0, M1, M2, and $\mathrm{HgSMs}$ as described above. THP-1 cell activation and polarization were performed in a $0.4-\mu \mathrm{m}$ cell inserts and cultured with hMADS adipocytes in the lower chamber. For controls, cells were cultured individually. Co-culture experiments were carried on for $48 \mathrm{~h}$.

SA- $\beta$-galactosidase staining for cell culture

SA- $\beta$-Gal activity was detected by using Senescence Detection Kit (Biovision). Briefly, M0 and $\mathrm{HgSM}$ cultured in 12-well plates were fixed for $15 \mathrm{~min}$ at room temperature and then washed twice in PBS. Cells were incubated overnight at $37^{\circ} \mathrm{C}$ with Staining Solution Mix (containing X-gal).

Cytokine releasing measurement

Condition media were collected at the end of each incubation, centrifuged, and stored at $-80^{\circ} \mathrm{C}$ until use in the assays. IL- 6 and IL- $1 \beta$ concentrations ( $\mathrm{pg} / \mathrm{ml}$ ) were measured using commercially available, highsensitivity ELISA kits (Cayman Chemical and Finetest) according to the manufacturer's instructions.

mRNA and mature miRNA quantitative RT-PCR

Total RNA was isolated using the Norgen Biotek Kit (Thorold, ON, Canada), according to the manufacturer's instructions. For adipose tissue, a first step with Trizol (ThermoFisher Scientific, Massachusetts, USA) and chloroform (Sigma-Aldrich) was performed to remove lipid content. RNA was stored at $-80{ }^{\circ} \mathrm{C}$ until use.

RNA amount was determined by spectrophotometric quantification with Nanodrop ONE (NanoDrop Technologies, Wilmington, DE, USA). Total RNA was reverse-transcribed using PrimeScript RT reagent Kit (Takara bio, Japan) according to the manufacturer's instructions. qRT-PCR was performed in a Rotor-Gene Q (Qiagen Hilden, Germany) using TB Green Premix Ex Taq (Takara bio, Japan). All primers (Table 2) were from Merck 
Table 2 Primers and TaqMan miRNA assays

\begin{tabular}{lll}
\hline Target gene & Forward & Reverse \\
\hline ADIPOQ & GAGATGGACGGACGGAGTCCTTTAGG & CTGGTCATGTTTGTGAAGCTCCC \\
$\beta$-actin & TGAGAGGGAAATCGTGCGTG & TGCTTGCTGATCCACATCTGC \\
CD206 & TATGGAATAAAGACCCGCTGAC & TGCTCATGTATCTCTGTGATGCT \\
GAPDH & GGCACAGTCAAGGCTGAGAATG & ATGGTGGTGAAGACGCCAGTA \\
GLUT4 & CATTCCTTGGTTCATCGTG & ATAGCCTCCGCAACATAC \\
IL-1 $\beta$ & AGATGATAAGCCCACTCTACAG & ACATTCAGCACAGGACTCTC \\
IL-6 & TGCAATAACCACCCCTGACC & GTGCCCATGCTACATTTGCC \\
IL-8 & GGACAAGAGCCAGGAAGAAA & CCTACAACAGACCCACACAATA \\
IL-10 & AGGCATTCTTCACCTGCTCC & AAGACCCAGACATCAAGGCG \\
MCP1 & GGCTGAGACTAACCCAGAAAAG & GGGTAGAAACTGTGGTTCAAGAG \\
NF-kB & ACAGCTGGATGTGTGACTGG & TCCTCCGAAGCTGGACAAAC \\
p21 & CCATCCCTCCCCAGTTCATT & AAGACAACTACTCCCAGCCC \\
SIRT1 & TGTTTCCTGTGGGATACCTGA & TGAAGAATGGTCTTGGGTCTTT \\
TNF- $\alpha$ & AAGCCTGTAGCCCACGTGTA & GGCACCACTAGTTGGTGGTCTTTG \\
TGF-b & CCCAGCATCTGCAAAGCT & GTCAATGTACAGCTGCCGCA \\
IL-1 $\alpha$ & CTTTCCCTGCCTGACCTTATT & GAATGAAGCTACTGCCCTACTC \\
PPAR-g & TGTGGGGATAAAGCATCAGGC & CCGGCAGTTAAGATCACACCTAT \\
CD163 & ACTGCAAGAACTGGCAATGG & CCATGCTTCACTTCAACAGG \\
miR-21 & TaqMan assay ID & \\
miR-146a & ID00397 & \\
RNU44 & ID000468 & \\
\hline & ID001094 & \\
\hline
\end{tabular}

Millipore (Darmstadt, Germany). Each single sample of the biological triplicate was loaded in a technical duplicate into the real-time plate. GAPDH and $\beta$-actin were used as endogenous controls. mRNA expression was assessed using the $2^{-\mathrm{DDCt}}$ method.

The expression of miR-146a and miR-21 (Table 2) was quantified by RT-qPCR using TaqMan miRNA assays (all from ThermoFisher
Scientific) according to the manufacturer's protocol. Data were analyzed with Rotor Gene Q (Qiagen, Hilden, Germany) with the automatic comparative threshold $(\mathrm{Ct})$ setting for adapting baseline. qRTPCR data were normalized to RNU44 (Table 2). The $2^{- \text {DDCT }}$ method was used to determine miRNA expression.
Table 3 Primary antibodies

\begin{tabular}{llll}
\hline Antibodies & Host* & Dilution & Source \\
\hline AKT & $\mathrm{R}$ & $1: 1000$ & Cell Signaling Technology/9272 \\
$\alpha$-tubulin & $\mathrm{R}$ & $1: 1000$ & Cell Signaling Technology/ 2144 \\
$\beta$-actin & $\mathrm{M}$ & $1: 200$ & Santa Cruz Biotechnology/sc-47778 \\
IKB- $\alpha$ & $\mathrm{R}$ & $1: 1000$ & Cell Signaling Technology/9242 \\
IL-1 $\beta$ & $\mathrm{R}$ & $1: 1000$ & Cell Signaling Technology/12242 \\
IRS1 & $\mathrm{M}$ & $1: 200$ & Santa Cruz Biotechnology/sc-8038 \\
JNK & $\mathrm{M}$ & $1: 200$ & Santa Cruz Biotechnology/sc-7345 \\
p21 & $\mathrm{R}$ & $1: 1000$ & Cell Signaling Technology/2947 \\
pAKT (Ser473) & $\mathrm{R}$ & $1: 2000$ & Cell Signaling Technology/4060 \\
pJNK & $\mathrm{M}$ & $1: 100$ & Santa Cruz Biotechnology/sc-6254 \\
SIRT1 & $\mathrm{R}$ & $1: 1000$ & Cell Signaling Technology/2496 \\
\hline
\end{tabular}


A

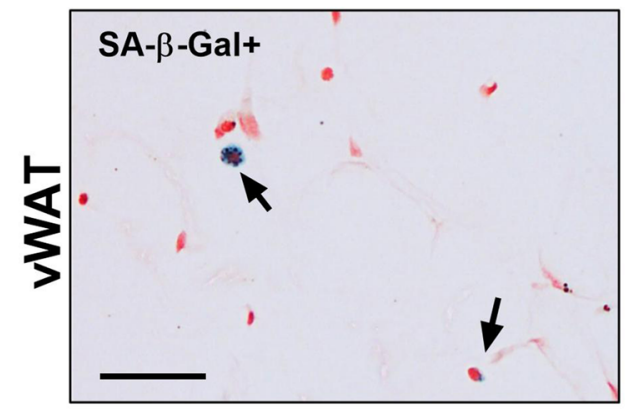

C

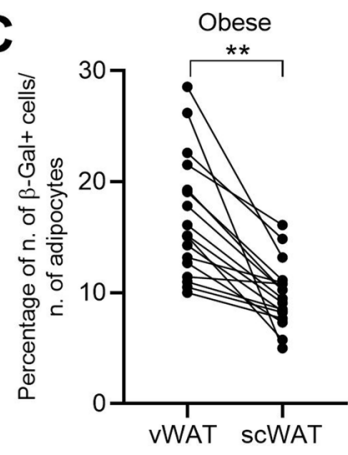

G
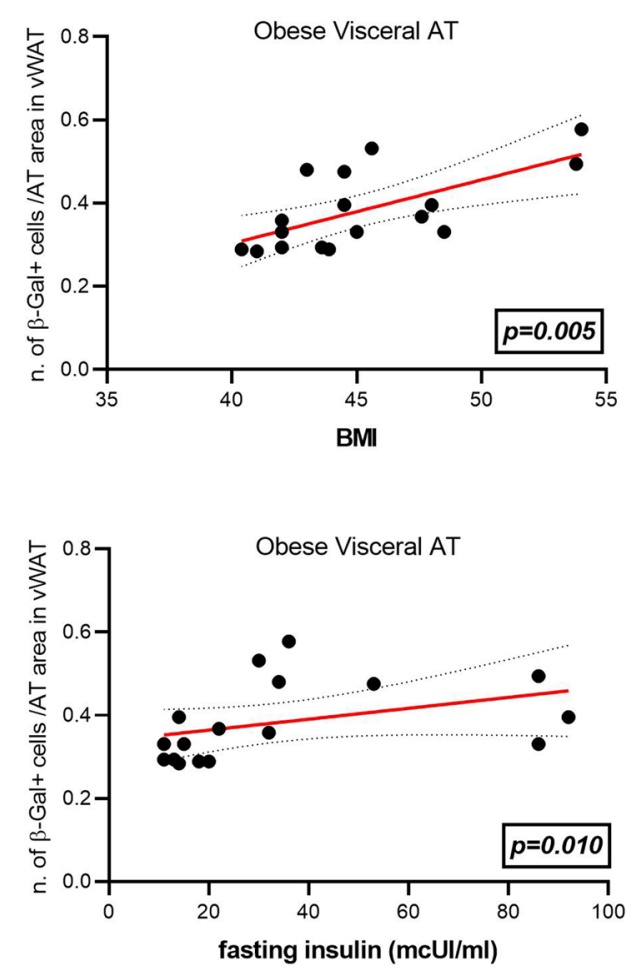

B

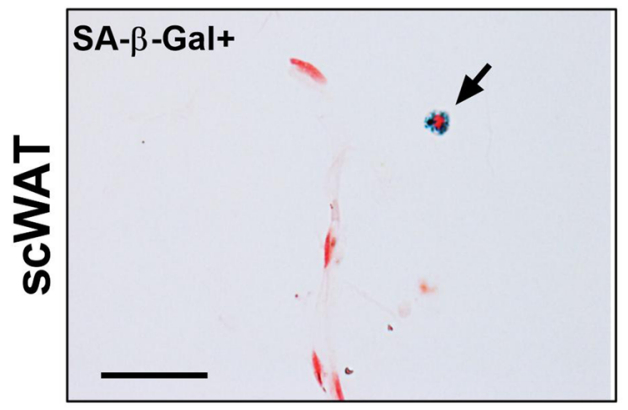

E
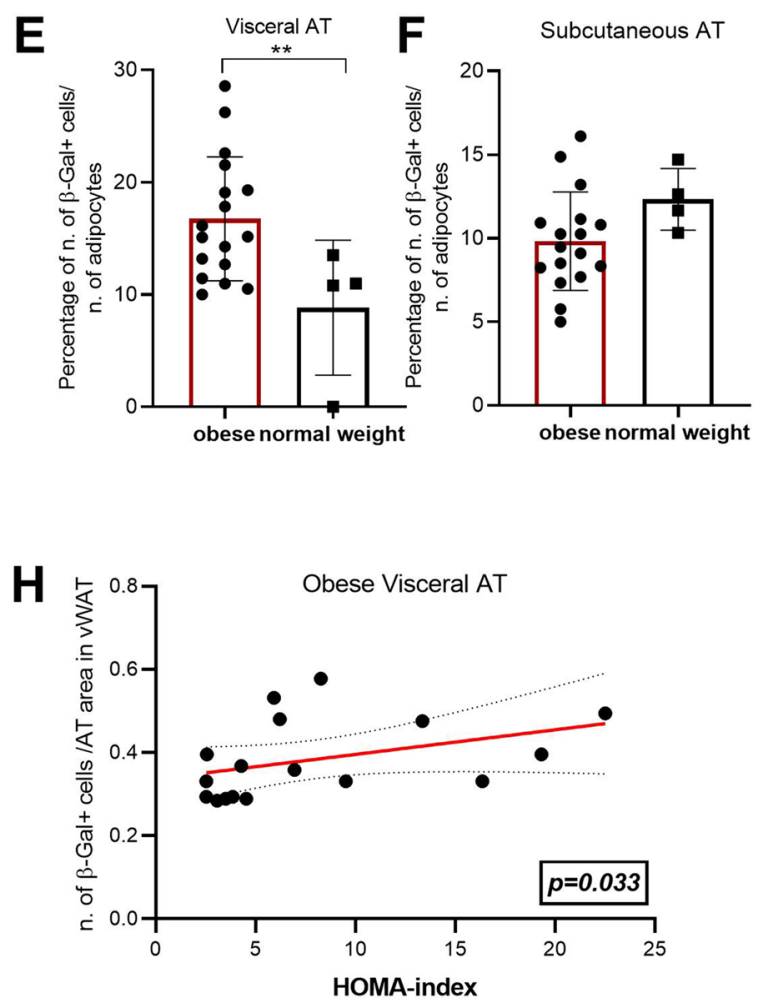
\Figure 1 SA- $\beta$-Gal + cells in adipose tissue. Histological analysis of visceral (vWAT) (A) and subcutaneous adipose tissue (scWAT) (B) of obese subjects $(n=17)$. Percentage of SA- $\beta-$ Gal + cells number in vWAT and scWAT in obese subjects $(\mathbf{C})$ and in normal weight subjects $(n=4)(\mathbf{D})$. Percentage of SA- $\beta$-Gal + cells number in vWAT between obese and normal weight subjects $(\mathbf{E})$. Percentage of SA- $\beta-$ Gal + cells number in scWAT between obese and normal weight subjects $(\mathbf{F})$. Spearman correlation between number of SA- $\beta$-Gal + cells and G BMI $(r=0.671 ; p=0.003)$, H HOMA index $(r=0.519$; $p=0.033)$, and $\mathbf{I}$ insulin $(r=0.607 ; p=0.010)$ in vWAT. Bar= $100 \mathrm{~mm} .{ }^{*} p \leq 0.05 ; * * p \leq 0.01 ; * * * p \leq 0.001$

DNA isolation and telomere length measurement

DNA was isolated using E.Z.N.A. Tissue DNA Kit (Omega bio-tek), according to the manufacturer's instructions. DNA was stored at $-80{ }^{\circ} \mathrm{C}$ until use. The abundance of telomere signal per genome measured by qPCR represents the average telomere length in a given DNA sample.

The amount of input genomic DNA is quantified by measuring the qPCR product of a single copy gene (S) that is used to normalize the signal from the telomere (T) reaction. The single-copy gene is the 36B4, which encodes acidic ribosomal phosphoprotein PO. The resulting T/S ratio represents the average telomere length per genome.

\section{Protein extraction and immunoblotting}

Cell lysates were obtained by using RIPA buffer (150 mM NaCl, $10 \mathrm{mM}$ Tris, pH 7.2, 0.1\% SDS, $1.0 \%$ Triton X-100, 5 mM EDTA, pH 8.0) containing a protease inhibitor cocktail (Roche Applied Science, Indianapolis, IN, USA). Protein concentration was determined using Bradford Reagent (Sigma-Aldrich). Total protein extracts (20 $\mu \mathrm{g}$ for hMADS and $15 \mu \mathrm{g}$ for THP-1) were separated by SDS-PAGE and transferred to nitrocellulose membranes (Bio-Rad, Hercules, CA, USA). Membranes were then blocked for $1 \mathrm{~h}$ at room temperature (RT) in TBS-Tween-20 containing $5 \%$ non-fat-dried milk and subsequently incubated overnight with the primary antibody (Table 3). Finally, they were incubated with a secondary antibody conjugated to horseradish peroxidase (Vector) for $1 \mathrm{~h}$ at $\mathrm{RT}$ and bands were visualized with the Chemidoc Imaging System (Bio-Rad) using ECL Plus chemiluminescence substrate (GE Healthcare, Pittsburgh, PA, USA). Densitometric analysis was performed with ImageJ software. When appropriate, membranes were stripped by incubation at room temperature for $7 \mathrm{~min}$ with a mild stripping buffer (15 $\mathrm{g}$ of glycine, $1 \mathrm{~g}$ of SDS, $10 \mathrm{ml}$ of Tween 20 and bring volume up to 11 with ultrapure water). They were then washed, blocked, and re-probed for total protein content.

Statistical analysis

Summarized data are shown as mean \pm SD or as frequency (\%). Independent sample $T$ test was used for the analysis of real-time and densitometric data. Paired $T$-test was used to compare vWAT and scWAT. Partial correlation, adjusted for age and gender, was used to test for correlations in the obese group. Correlations between parameters were calculated using Spearman's rho. Chi-square test was used as appropriate.

Data analysis was performed using IBM SPSS Statistics for Windows, version 25 (IBM Corp, Armonk, NY, USA). Statistical significance was defined as a two-tailed $p$-value $<0.05$.

\section{Results}

Accumulation of SA- $\beta-\mathrm{Gal}+$ macrophages in vWAT from obese patients

SCs can be identified by elevated lysosomal SA- $\beta$ Gal activity. Here, SA- $\beta$-Gal positive $(+)$ cells were scored in visceral (vWAT, Fig. 1A) and subcutaneous (scWAT, Fig. 1B) adipose tissues of obese individuals underwent bariatric surgery $(n=17)$. A significantly higher number of SA- $\beta$-Gal + cells were observed in vWAT compared to scWAT from the same obese subject (Fig. 1C). Conversely, when SA- $\beta-$ Gal + cells were evaluated in a normal weight control group $(n=4)$, we did not detect significant difference in the number of SA- $\beta-\mathrm{Gal}+$ cells in vWAT compared to scWAT (Fig. 1D). Notably, the comparison between obese patients and normal weight controls revealed a significantly greater amount of SA- $\beta-$ Gal + cell in vWAT of obese individuals compared to controls (Fig. 1E), whereas no significant difference was found in scWAT of obese individuals compared to controls (Fig. 1F). 
In obese patients, SA- $\beta-$ Gal + cell number was positively correlated with BMI in vWAT but not in scWAT samples (Fig. $1 \mathrm{G}$ and Suppl. Fig. 1(i)). Importantly, in vWAT, SA- $\beta$-Gal + cell number was positively correlated also with the most clinically relevant markers of insulin resistance, such as HOMA index and insulin levels (Fig. 1H, I) but not in scWAT samples (Suppl. Fig. 1 (ii) and (iii)).

The correlations between SC number in vWAT of obese subjects and some parameters relevant in the management of diabetic patients prompted us to unravel the phenotype of senescent cells. vWAT and scWAT tissues were assessed by immunohistochemical analysis with the pan-macrophage marker CD68: the majority of SCs was positive for CD68 (Fig. 2A, B). In particular, the percentage of the double positive (SA- $\beta-\mathrm{Gal} / \mathrm{CD} 68+$ ) cells on the total amount of SA- $\beta$-Gal + cells was $69.75 \%$ in vWAT and $69.25 \%$ in scWAT of obese individuals (Fig. 2C). Moreover, an additional marker of senescence, $\mathrm{p} 16^{\mathrm{INK} 4 \mathrm{a}}$, was performed in vWAT and scWAT of obese subjects by immunohistochemical analysis. A consistent number of $\beta$-Gal + cells resulted immunoreactive for the marker $16^{\mathrm{INK} 4 \mathrm{a}}$. In Supplementary Figure 2 is shown a crown-like structure (CLS) characterized by aggregates of macrophages surrounded dead adipocytes [28].

Overall, these results suggest that the accumulation of senescent macrophages in visceral adipose tissue is related to the presence of insulin resistance in obese individuals.

THP-1 cells cultured in high glucose medium show senescent features and mixed M1/M2 phenotype

To model senescent macrophages in vitro, we took advantage of the THP-1-derived macrophage model. THP-1 cells were differentiated into naïve (M0) macrophages by PMA to obtain the control condition, then polarized into M1 (by LPS) and M2 (by IL-4 and IL-13) macrophages. To mimic the exposure to hyperglycemia, THP-1 cells were cultured in the presence of high glucose concentration $(60 \mathrm{mM})$ for 1 week and then induced with PMA to macrophages, henceforth named high-glucose senescent macrophages (HgSM). Morphological features of the different phenotypes were observed by optical microscopy (Fig. 3A). First, we observed no significant difference in proliferative activity and viability of
THP-1 cells cultured in high-glucose medium compared to normoglycemic condition (Fig. 3B, C).

Thus, independently from proliferation, we found several senescence biomarkers in HgSMs. Namely, SA- $\beta$-Gal activity (Fig. 3D) and CDK p21 expression (Fig. 3E) were significantly higher in $\mathrm{HgSMs}$ than in M0 macrophages. Moreover, telomere length (Fig. 3F) and SIRT1 expression (Fig. 3G) were reduced in $\mathrm{HgSMs}$ compared to M0 macrophages.

A number of M1 and M2 markers were analyzed in THP-1-derived macrophage, showing the appropriate differentiation of M1 and M2 macrophages (upregulation of IL-1a and NF-kB (p65), TNF-a, IL-8 and IL-6 for M1 and PPAR-g, TGF-b and CD163 for M2) (Fig. 4A, B).

Notably, HgSMs also showed a peculiar pattern of M1/M2 marker expression, dissimilar to M1- and M2-differentiated THP-1 cells, compared to controls (Fig. 4A, B). Indeed, HgSM cells expressed the highest level of NF-kB and PPAR-g as well as the lowest level of CD163 compared to M0, M1, and M2 macrophages (Fig 4A). Moreover, HgSMs conveyed a concomitant increase in pro-inflammatory TNF- $\alpha$ and anti-inflammatory IL-10 mRNA cytokine, coupled with a reduced expression of IL-8, IL-6, and IL-1 $\beta$ (Fig. 4B). Data on the low expression of IL-1 $\beta$ and IL-6 in HgSMs were confirmed at protein level by ELISA for both cytokines (Fig. 4C) and by Western blot analysis for IL-1 $\beta$ (Fig. 4D).

Collectively, our data show that high glucose triggers a senescent phenotype with M1/M2 intermediate features in THP-1- derived macrophages.

Interplay between $\mathrm{HgSM}$ macrophages and hMADS adipocytes fosters inflammation

To disentangle the dynamic interplay between macrophages and adipocytes in vitro, we set up a coculture system of THP-1-derived M0, M1, M2, and $\mathrm{HgSM}$ macrophages and differentiated hMADS adipocytes. These latter after 12 to 15 days of differentiation show an abundant multilocular lipid content that can be easily visualized by oil red $\mathrm{O}$ staining (Suppl Fig. 3A) and express the typical markers of mature white adipocytes [27]. THP-1 monocytic cells were seeded onto transwell inserts, differentiated into M0, M1, M2, and HgSM macrophages, then co-cultured with differentiated hMADS adipocytes for 48 h (Suppl Fig. 3B). Real-time PCR analysis of HgSM 
Figure 2 Senescent macrophages in obese adipose tissue. Immunohistochemical analysis of SA- $\beta-\mathrm{Gal}+$ / CD68+ cells in vWAT (A) and scWAT (B) of obese individuals. C Percentage of the double positive $(\mathrm{SA}-\beta-\mathrm{Gal}+/ \mathrm{CD} 68+$ ) cells in vWAT and scWAT of obese subjects compared to the total amount of SA- $\beta$ $\mathrm{Gal}+$ cells. Bar $=100 \mathrm{~mm}$. $* p \leq 0.05$; * $p \leq 0.01$; $* * * p \leq 0.001$
A

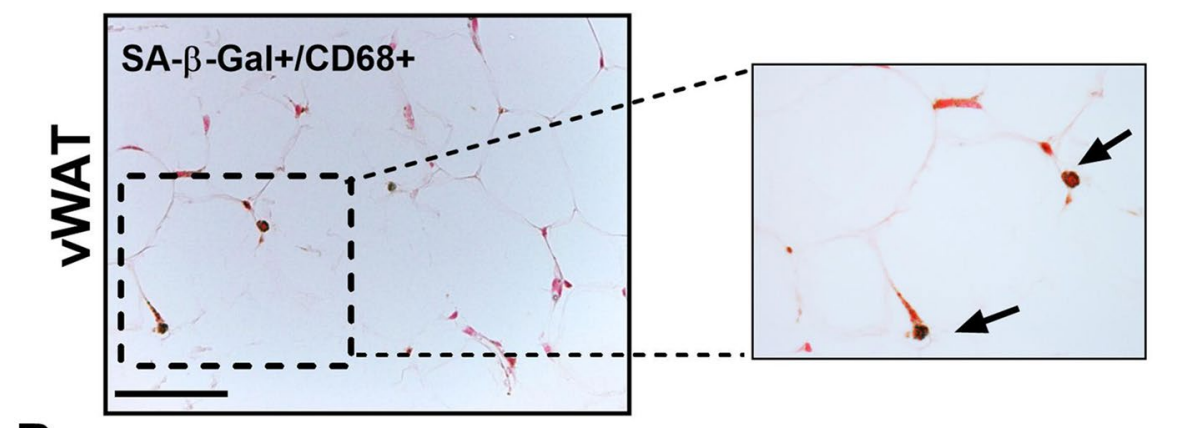

B
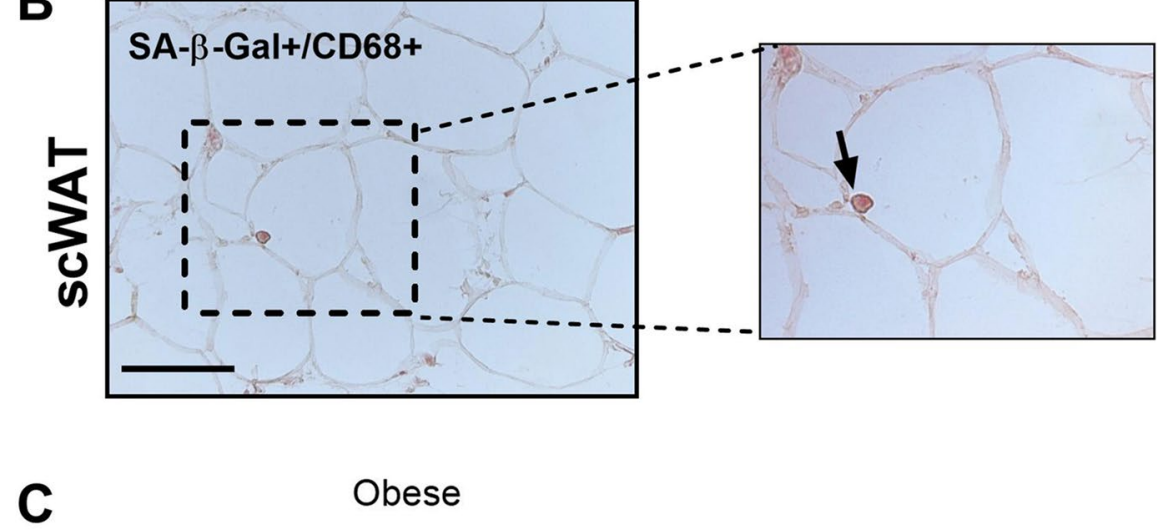
Obese

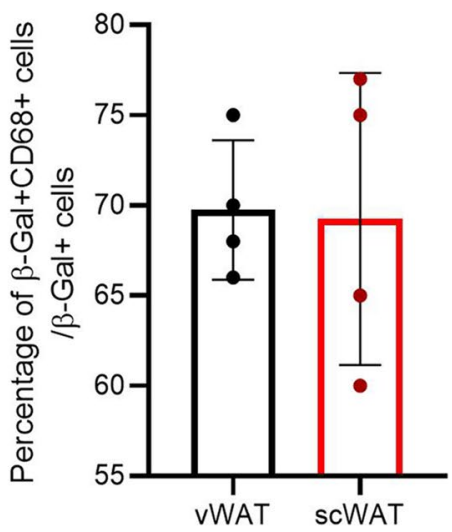

cells co-cultured with hMADS adipocytes showed a significantly higher expression of pro-inflammatory cytokines TNF $\alpha$, IL-8, and IL-6, together with a significantly reduced expression of IL-10 mRNA, in comparison to M0 cells (Fig. 5A). As expected, M1 macrophages showed the typical increased expression of IL-6 mRNA together with low IL-10 mRNA level (Fig. 5A). IL-1 $\beta$ protein expression was significantly increased in $\mathrm{HgSMs}$ co-cultured with hMADS (Fig. 5B). Since the most relevant proinflammatory cytokines are produced under the control of the NF-kB proinflammatory pathway, we analyzed IKB- $\alpha$ protein, inhibitor of NF-kB signal [29]. A significantly reduced expression of IKB- $\alpha$ protein was observed in HgSMs compared to M0 cells (Fig. 5B), as well as in hMADS adipocytes co-cultured with HgSMs (Fig. 5C). An increased mRNA expression of the p65 NF-kB subunit was observed in hMADS co-cultured with all the macrophage phenotypes (Suppl.Fig. 3C). Overall, these results suggest that $\mathrm{HgSMs}$ can worsen the pro-inflammatory activity induced by NF-kB signaling activation in adipocytes, 
A

THP1

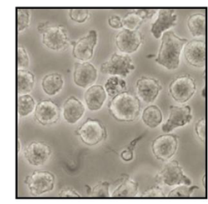

B

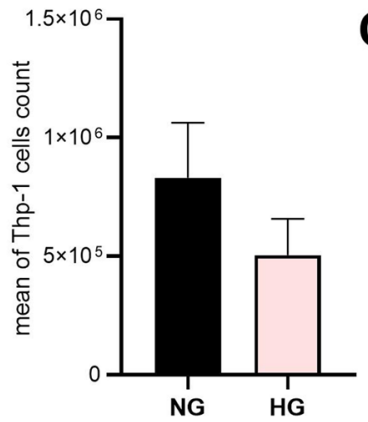

$\mathbf{E}$

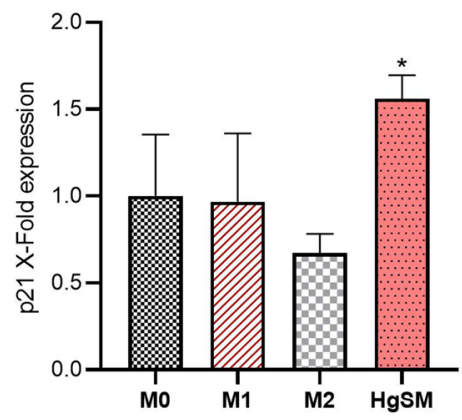

MO

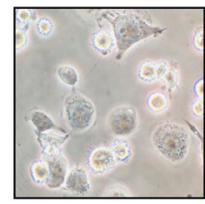

M1

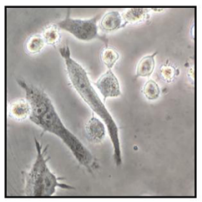

M2

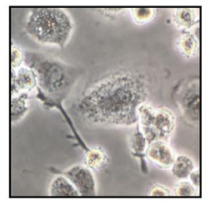

HgSM

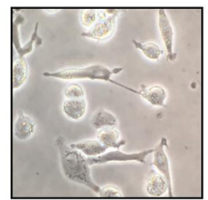

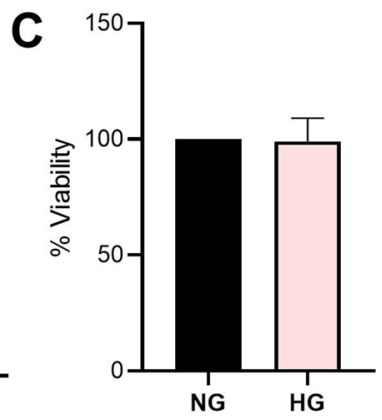

$\mathbf{F}$
D
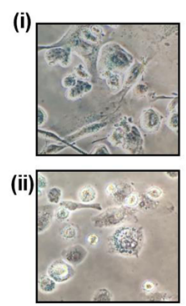

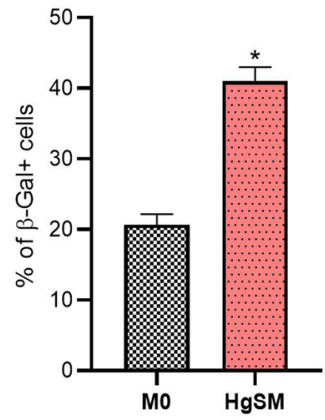

G

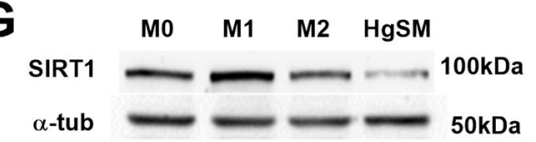

Figure 3 Analysis of PMA-induced macrophage phenotypes and senescent features in $\mathrm{HgSMs}$. A Representative pictures from THP-1 cells and M0, M1, M2, and HgSM macrophage polarization observed by light microscopy $(40 \times)$. B THP-1 proliferative activity in hyperglycemia compared to normoglycemia. C THP-1 viability in hyperglycemia compared to normoglycemia performed by MTT assay. D SA- $\beta$-Gal activity of
HgSMs (i) compared to M0 (ii). E p21 qRT-PCR analysis in M0, M1, M2, and HgSMs. F Measurements of telomere length (T/S ratio) in M0, M1, M2, and HgSM macrophages. G Representative immunoblot and quantification of SIRT1 expression in M0, M1, M2, and HgSM macrophages. Data are expressed as mean $\pm \mathrm{SD}, * p \leq 0.05 ; * * p \leq 0.01 ; * * * p \leq 0.001$ a condition that could be observed in vivo when macrophages infiltrate adipose tissue. In accordance with this hypothesis, hMADS adipocytes co-cultured with $\mathrm{HgSM}$ macrophages showed a significant hyperexpression of the pro-inflammatory cytokines IL-6, IL-1 $\beta$, TNF- $\alpha$, IL- 8 , and MCP1 together with a significant downregulation of adiponectin (ADIPOQ) mRNA compared not only to hMADS adipocytes cultured alone (control) but also to hMADS co-cultured with M0 cells (Fig. 5D and Fig. 5E). In accordance, hMADS adipocytes co-cultured with $\mathrm{HgSMs}$ secreted a significative higher concentration of IL- $1 \beta$ compared to hMADS adipocytes co-cultured with M0 (Fig. 5F).

A reduced SIRT1 mRNA expression and protein levels was observed in hMADS co-cultured with all 
A

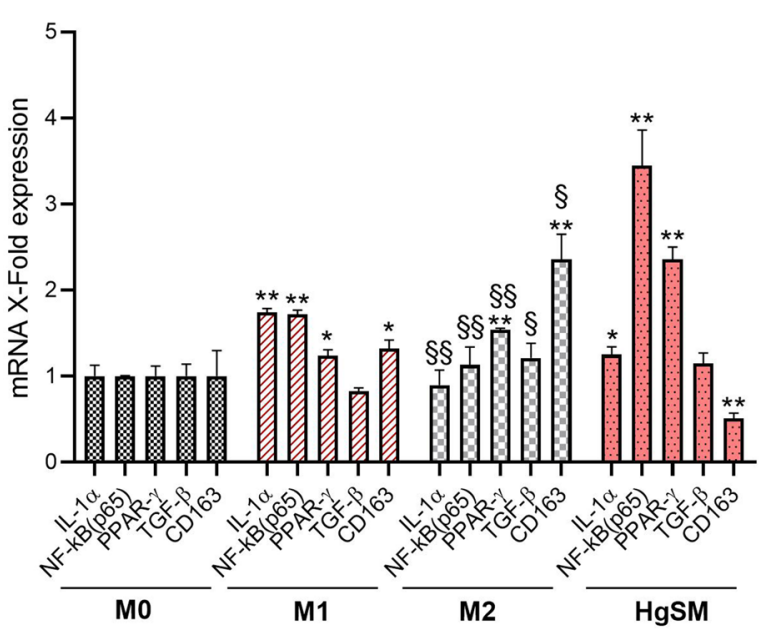

C
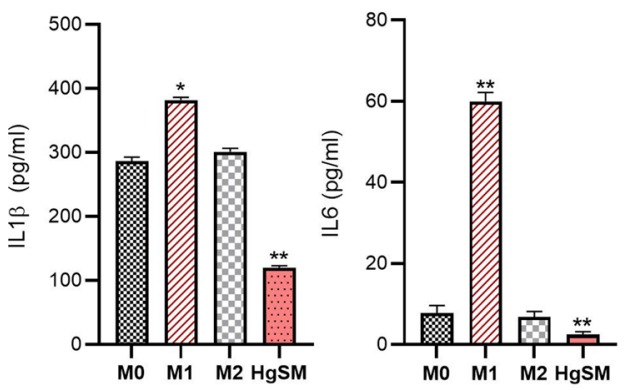

Figure 4 Phenotypic characterization of $\mathrm{HgSM}$ macrophages. A qRT-PCR analysis of NF-kB, Il-1a, TGF-b, PPAR-g, and CD163 expression in macrophages. B qRT-PCR analysis of TNF- $\alpha$, IL-8, IL-6, IL-1 $\beta$, and IL-10 expression in macrophages. C IL-1 $\beta$ and IL- 6 concentration $(\mathrm{pg} / \mathrm{ml})$ measured

the macrophage phenotypes compared to the control adipocytes (Suppl Fig. 3C and Suppl Fig. 3D).

Finally, since C-Jun NH (2)-terminal kinase (JNK) signaling pathway is involved in the modulation of the inflammatory process and in the metabolic response to obesity, including insulin resistance [30], we analyzed THP-1-derived macrophages for JNK phosphorylation by Western blotting. Our analysis showed that, on one hand, only $\mathrm{HgSM}$ cells co-cultured with hMADS adipocytes exhibited increased JNK phosphorylation

B
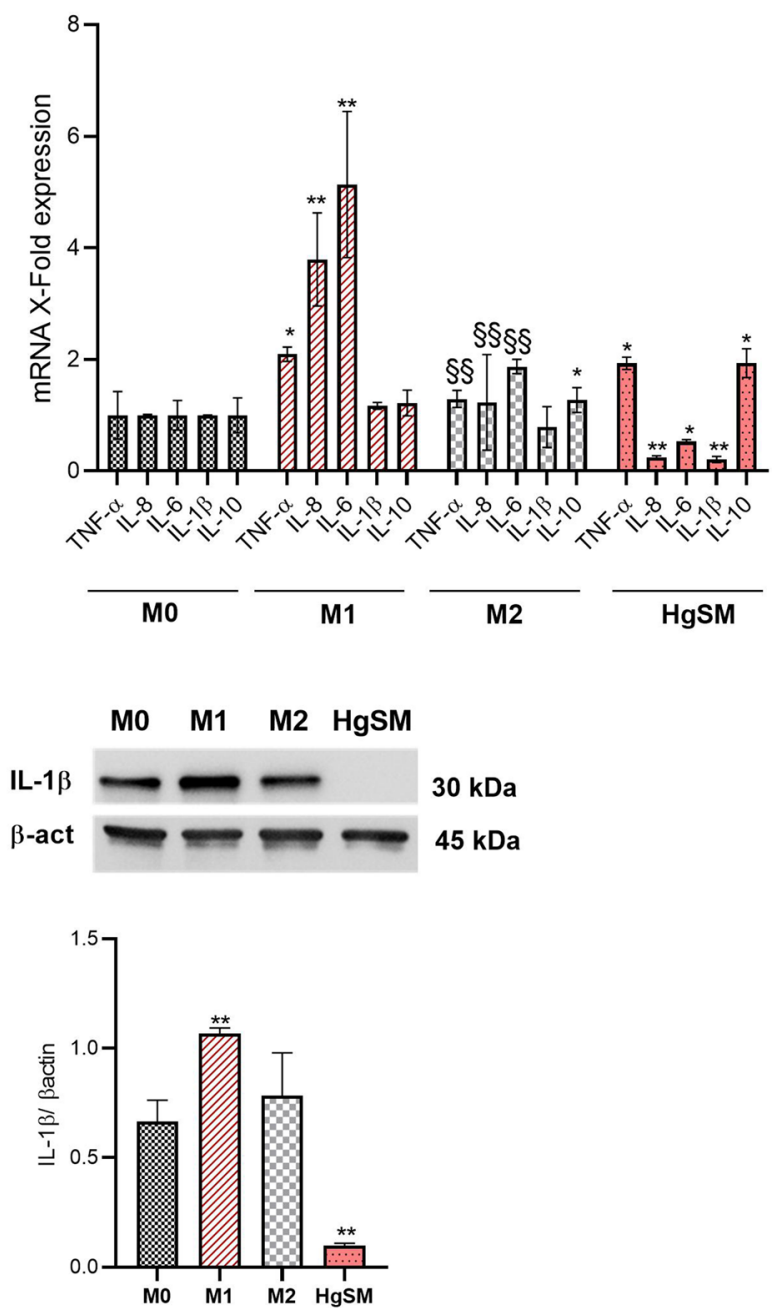

by ELISA in the cell culture supernatants. D Representative immunoblot and quantification of IL- $1 \beta$ expression in M0, M1, $\mathrm{M} 2$, and $\mathrm{HgSM}$ macrophages. Data are expressed as mean \pm $\mathrm{SD},{ }^{*} p \leq 0.05 ; * * p \leq 0.01 ; * * * p \leq 0.001 ;{ }^{\S} p \leq 0.05 ;{ }^{\S} p \leq 0.01$; ${ }^{\S \S} p \leq 0.001$ * *v M0, ${ }^{\S} \mathrm{vs}$ M1

compared to M0 cells (Fig. 5G). On the other hand, hMADS co-cultured with M0, M1, M2, and $\mathrm{HgSM}$ macrophages exhibited increased JNK phosphorylation compared to the control hMADS (Suppl Fig. 3E). Collectively, these data suggest that the co-culture of $\mathrm{HgSM}$ macrophages and hMADS adipocytes (1) moves senescent $\mathrm{HgSMs}$ toward a pro-inflammatory phenotype, and (2) moves hMADS adipocytes toward a proinflammatory phenotype, characterized by reduced 


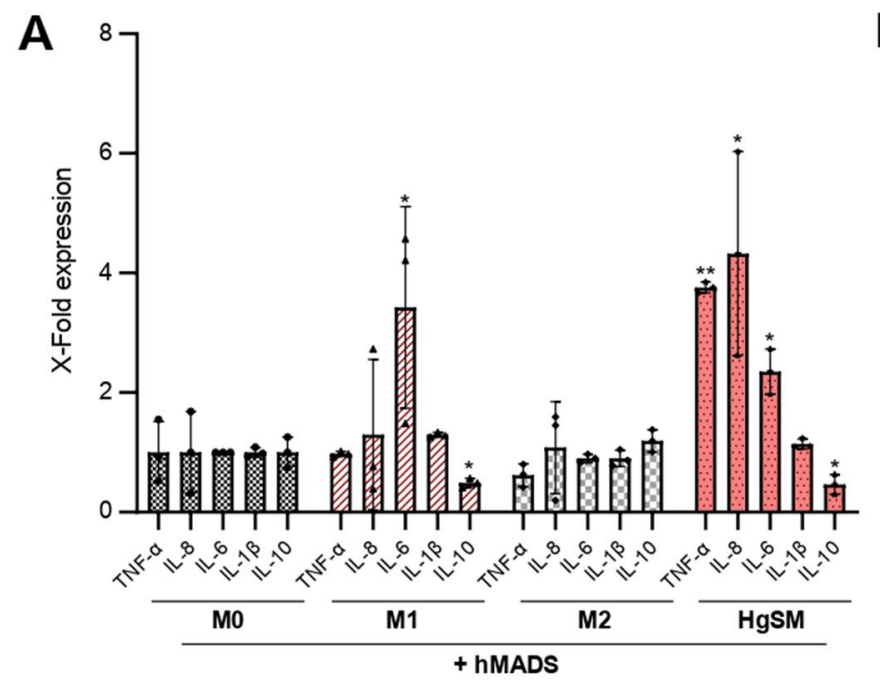

B
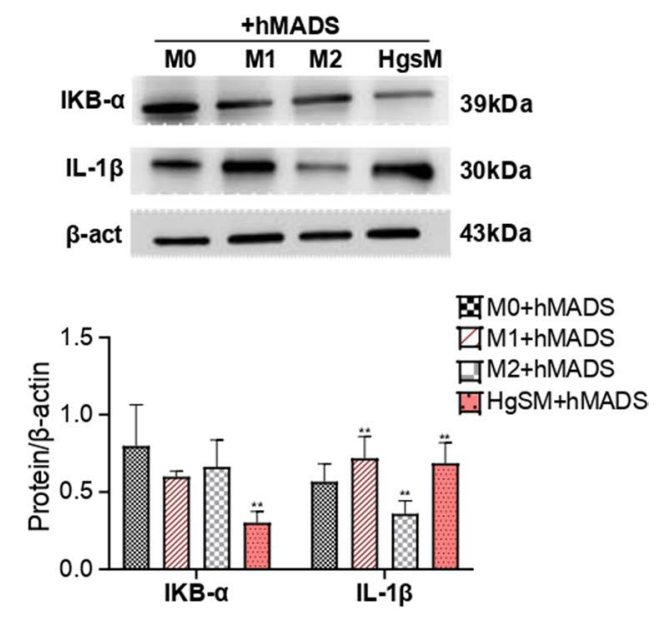

C

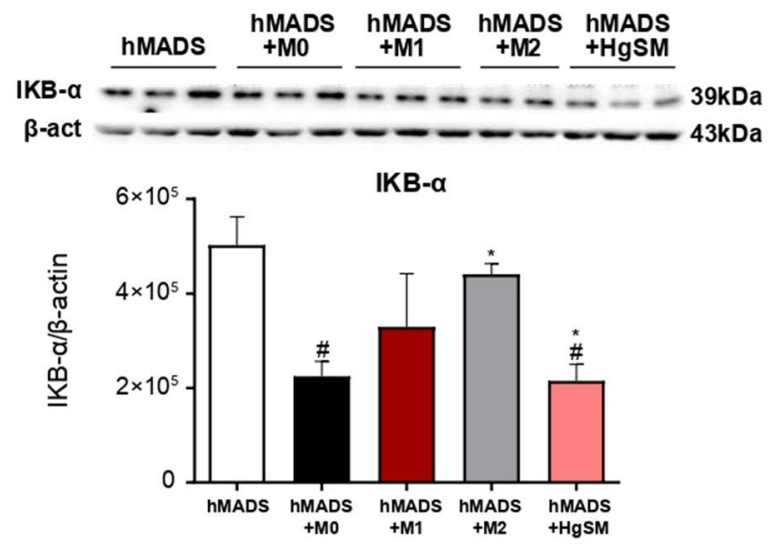

D
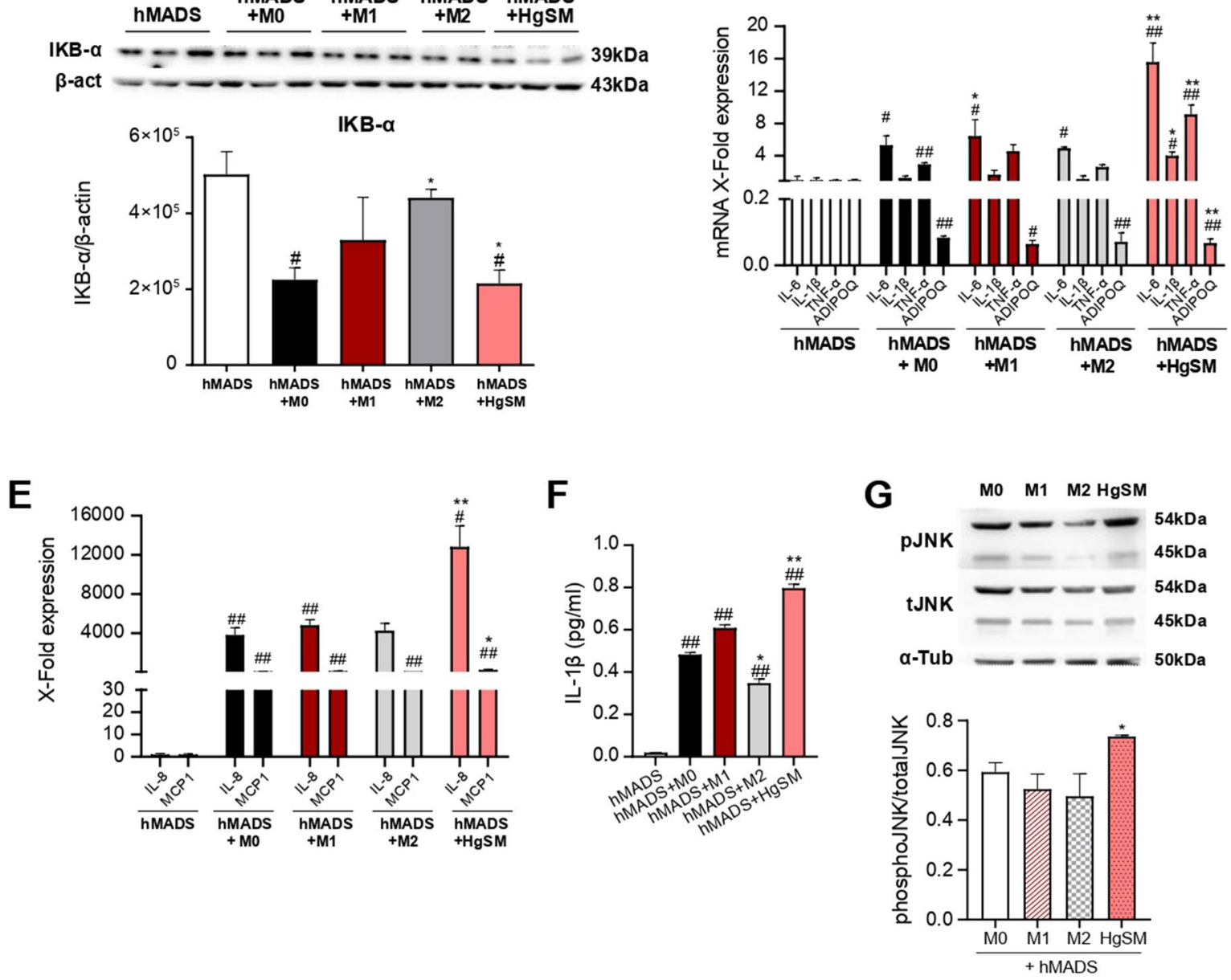
4Figure 5 Phenotypic characterization of HgSM macrophage and hMADS adipocyte co-culture. A qRT-PCR analysis of TNF- $\alpha$, IL-8, IL-6, IL-1 $\beta$, and IL-10 expression in macrophages co-cultured with hMADS adipocytes. B Representative immunoblot and quantification of IKB- $\alpha$ and IL- $1 \beta$ in macrophages co-cultured with hMADS adipocytes. C Representative immunoblot and quantification of IKB- $\alpha$ in hMADS adipocytes co-cultured with macrophages. qRT-PCR analysis of IL-6, IL-1 $\beta$, TN-F $\alpha$, ADIPOQ D, IL-8, and MCP1 E expression in hMADS adipocytes co-cultured with macrophages. F IL-1 $\beta$ concentration $(\mathrm{pg} / \mathrm{ml})$ measured by ELISA in cell co-culture supernatant. G Representative immunoblot and quantification of pJNK expression in macrophages cocultured with hMADS adipocytes. Data are expressed as mean $\pm \mathrm{SEM}$ or mean $\pm \mathrm{SD}$. $* p \leq 0.05 ; * * p \leq 0.01 ; * * * p \leq 0.001$; ${ }^{\#} p \leq 0.05 ;{ }^{\# \#} p \leq 0.01 ;{ }^{\# \#} p \leq 0.001$. *vs M0; \#vs hMADS

expression of adipokines and increased release of cytokines.

Effect of $\mathrm{HgSM}$ macrophages on insulin signaling in hMADS adipocytes

There is a close and bidirectional link between obesity, chronic inflammation, and insulin resistance. Adipose tissue inflammation, involving increased infiltration of M1 pro-inflammatory macrophages, adipocyte death and abnormal cytokine and adipokine secretion, is a major actor of obesity-induced chronic systemic inflammation and insulin resistance [31]. On the other hand, adipose tissue is an important target of insulin and an impairment of insulin signaling in subcutaneous and visceral fat importantly worsen body insulin sensitivity. Thus, we assessed the effects of $\mathrm{HgSM}$ macrophages on hMADS adipocyte insulin signaling by measuring the IRS 1 protein levels and the phosphorylation of the 473serine kinase AKT (pAKT), a central player in the insulin signaling pathway [32].

Western blot analysis demonstrated that hMADS exhibited a significantly reduced IRS1 expression in each experimental condition that was evident in HgSM-hMADS co-cultures (Fig. 6A).

With the purpose of analyzing the pAKT insulindependent signaling, hMADS adipocytes were cocultured with M0, M1, M2, and HgSM macrophages for $48 \mathrm{~h}$, and then incubated with $100 \mathrm{nM}$ insulin for $20 \mathrm{~min}$ before cell lysis, followed by the analysis of pAKT expression. Importantly, $\mathrm{HgSMs}$ induced a variable but significant reduction of pAKT level in response to $100 \mathrm{nM}$ insulin. The pAKT insulindependent signaling was also downregulated in M0-, M1-, and M2-treated hMADS adipocytes in comparison with insulin-treated hMADS adipocytes (Fig. 6B).

Finally, GLUT4 mRNA levels were examined. As shown in Fig. 6C, a notable reduction in GLUT4 levels was observed in hMADS adipocytes co-cultured with M0, M1, M2, and HgSM macrophages. Of note, HgSM-co-cultured adipocytes displayed a lower GLUT4 expression compared to M0 co-cultured adipocytes (Fig. 6C). Taken together, these results suggest that macrophages may functionally regulate insulin signaling and that $\mathrm{HgSM}$ may play a critical role in decreasing insulin signal transduction in human adipocytes.

MiRNAs expression in adipose tissues samples, in HgSMs and hMADS

To explore if the observed senescent phenotype of the adipose tissue of obese subjects is paralleled also by the rearrangement of miRNAs deregulated by senescence, we measured the expression levels of miR146a and miR-21 in the same adipose tissue samples of obese subjects, and in cellular models.

In adipose tissue samples, we observed a significant increased level of miR-146a in vWAT compared with scWAT (Fig. 7A). Further, miRNA expression analysis in macrophages differentiated in M1, M2, and $\mathrm{HgSM}$ confirmed a significant hyperexpression of miR-146a in M1 compared to M0, underlining the pro-inflammatory condition of M1 (Fig. 7B). In HgSMs, miR-21 levels were significantly reduced compared to M0 (Fig. 7B), whereas in HgSMs cocultured with hMADS adipocytes the expression levels of miR-21 were significantly increased (Fig. 7C).

Finally, adipocytes co-cultured with all macrophage phenotypes expressed higher level of miR146a, whereas only $\mathrm{HgSMs}$ induce a low but significant increase of miR-21 compared to control adipocytes (Fig. 7D).

Overall, these results suggest that miR-146a is hyperexpressed in vWAT samples, confirming its role as anti-inflammatory miRNA that attempts to restrain a proinflammatory condition.

In the cellular model, the co-culture of HgSMs with hMADS adipocytes is associated with an increased 
Figure 6 Effect of macrophages on insulin signaling in hMADS adipocytes. Representative immunoblot and quantification of IRS1 (A) and pAKT (B) in hMADS adipocytes cocultured with macrophages. C qRT-PCR analysis of GLUT4 expression in hMADS adipocytes cocultured with macrophages. Data are expressed as mean $\pm \mathrm{SEM}, * p \leq 0.05$ $* * p \leq 0.01 ; * * * p \leq 0.001$; ${ }^{\#} p \leq 0.05 ;{ }^{*} p \leq 0.01$; ${ }^{\# \# \#} p \leq 0.001,{ }^{\$} p \leq 0.05$; ${ }^{\$} p \leq 0.01 ;{ }^{\$} \$ p \leq 0.001$. *vs M0; \#vs hMADS; \$vs hMADS+Insulin
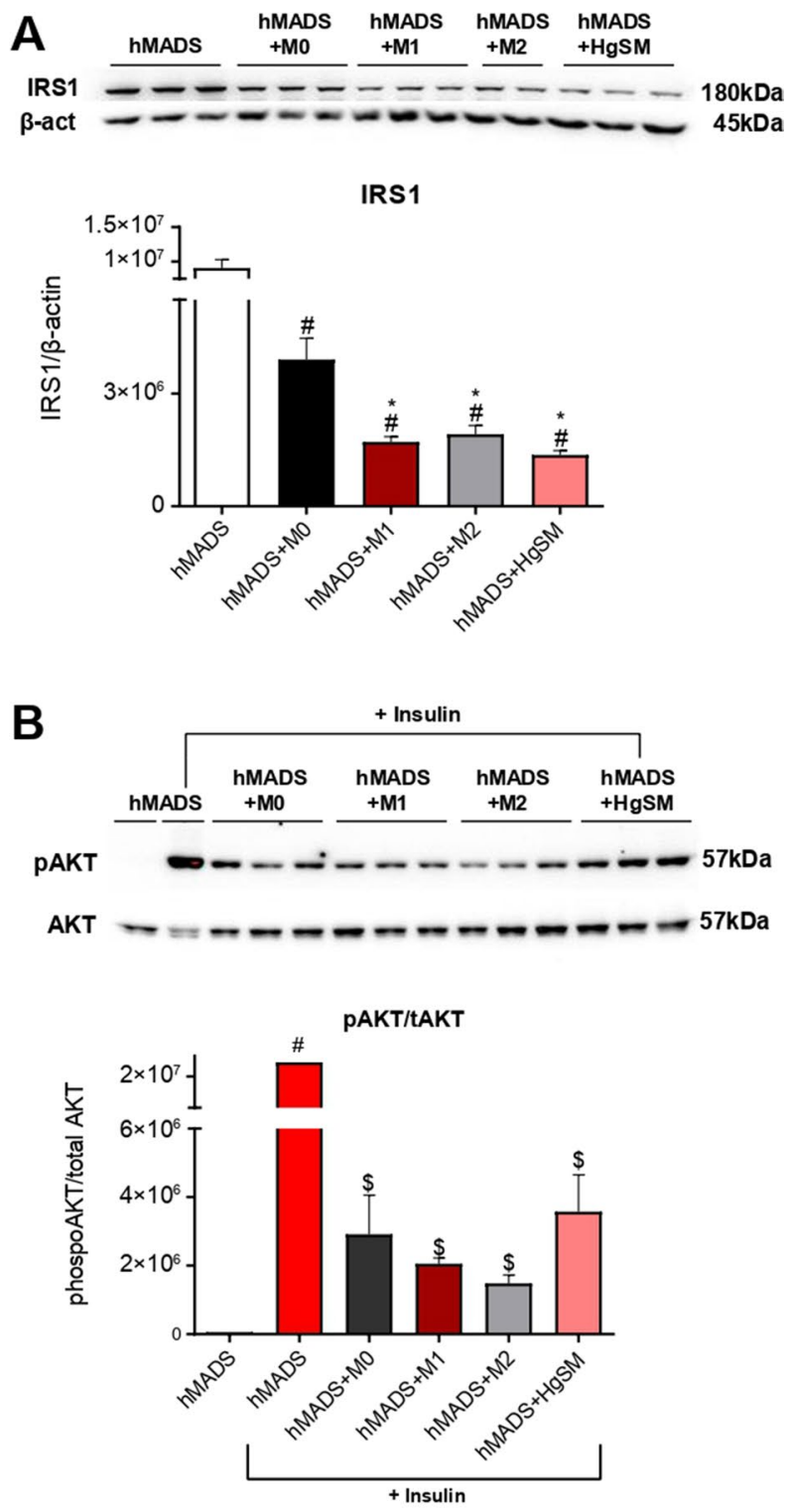

C

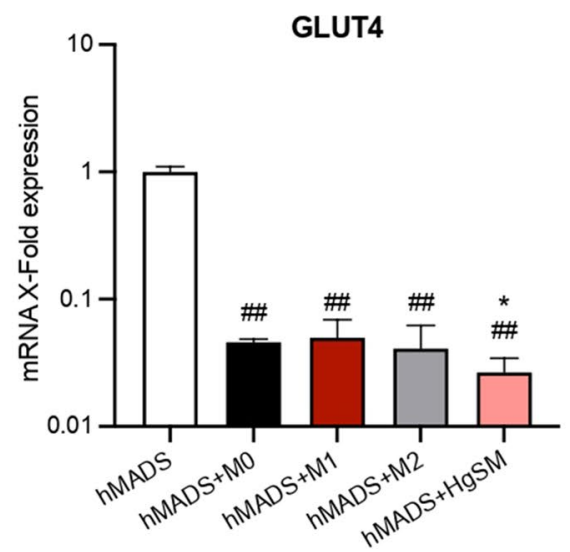


expression level of miR-21 in $\mathrm{HgSMs}$ as well as a higher level of miR-21 and miR-146a in hMADS.

These data confirmed the pro-inflammatory crosstalk between macrophages and adipocytes.

\section{Discussion}

Obesity is a powerful risk factor for T2DM and a major source of chronic and systemic inflammation [33]. Increasing evidence strongly points at the involvement of SCs in fueling inflammation and unbalancing insulin sensitivity [21]. In this investigation, a significant higher number of SA- $\beta$-Gal + cells was observed in vWAT compared to scWAT of obese patients. Moreover, SA- $\beta-\mathrm{Gal}+$ cells number in vWAT but not in scWAT was positively correlated with BMI, HOMA index, and serum insulin level. Owing to the role of inflammation in insulin resistance [34] and due to the expected role of senescencerelated secretome in systemic inflammation [35], it can be speculated that the accumulation of SCs within visceral adipose tissue depots might trigger insulin resistance and promote T2DM. Of note, the evaluation of SA- $\beta-\mathrm{Gal}+$ cells in a small group of normalweight individuals has revealed that the SA- $\beta-\mathrm{Gal}$ + cells were significantly lower in vWAT of normal weight controls compared to obese patients.

Such data suggest that the accrual observed in obese subjects might be of pathological relevance.

We also observed that the majority of SCs (about $70 \%$ of the total) in vWAT and scWAT were positive for the pan-macrophage marker CD68. Pre-adipocytes and $\mathrm{T}$ cells may concur to the $30 \%$ of SA- $\beta$ $\mathrm{Gal}+$ cells negative for CD68 in obese individuals, as suggested by previous studies [25, 36]. Senescent status of SA- $\beta$-Gal + cells was also confirmed by $\mathrm{p} 16^{\mathrm{INK} 4 \mathrm{a}}$ nuclear staining. Indeed, SCs have been analyzed in human abdominal and femoral subcutaneous fat biopsies from healthy subjects, showing an increased number of SCs in the subcutaneous abdominal adipose tissue, and an association with inflammatory signals [37]. Another study found an increased abundance of senescence markers in mesenchymal stromal cells harvested from subcutaneous fat tissue of obese subjects when compared to lean controls [38]. In addition, one report suggested that SCs present in thigh fat are associated with physical function [39]. Of note, SCs present in adipose tissue are emerging as druggable targets, given the ability of selected drugs to promote their clearance both in vivo and in ex vivo explants [40].

As adipose tissue composition is heterogeneous, the individual role of senescent cells within this tissue is debated [9, 41]. Preadipocytes can senesce, either via proliferative exhaustion during aging or by increased oxidative stress promoted by obesity [22, 42]. Interestingly, also chronic hyperinsulinemia has been recently suggested to drive adipocyte senescence through cell cycle re-entry [43]. Endothelial cells are present in the WAT vascular system and have been also suggested to acquire a senescent phenotype in obese subjects [44]. The vWAT of obese and old mice and humans is also enriched in immune cells with characteristics of cellular senescence, as $\mathrm{T}$ cells, the pro-inflammatory $\mathrm{ABCs}$, and macrophages [45]. Interestingly, also peripheral immune cells from patients with either obesity or T2DM are characterized by an accelerated senescent phenotype, extending the observations obtained in the adipose tissue at the systemic level [46, 47].

To our knowledge, our study, for the first time, conveys the presence of senescent macrophages within human vWAT and scWAT samples from the same subject. Notably, macrophages expressing senescence markers have been previously found to physiologically occur in the adipose tissue of aged mice [26, 48, 49]. However, it has been hypothesized that senescent macrophages can fuel inflammaging [50]. Since we recently have demonstrated a direct link between hyperglycemia and the senescent-associated secretory phenotype (SASP) in endothelial cells and macrophages [10], in this study, we aimed to characterize the senescence status acquired by macrophages in high-glucose milieu, and how it can be modulated by adipocyte crosstalk. After a week of high-glucosemedium exposure, a senescent phenotype in THP1-derived macrophages and a polarization toward a mixed M1/M2-like phenotype were observed. We called this peculiar macrophage phenotype as highglucose-induced senescent macrophage ( $\mathrm{HgSM})$ and we speculated that this may represent a phenomenon that occurs in vivo when macrophages are exposed to hyperglycemia. $\mathrm{HgSMs}$ are characterized by the simultaneous increased release of inflammatory and anti-inflammatory mediators, such as TNF- $\alpha$ and IL-10, indicative of a condition that has been termed 
A

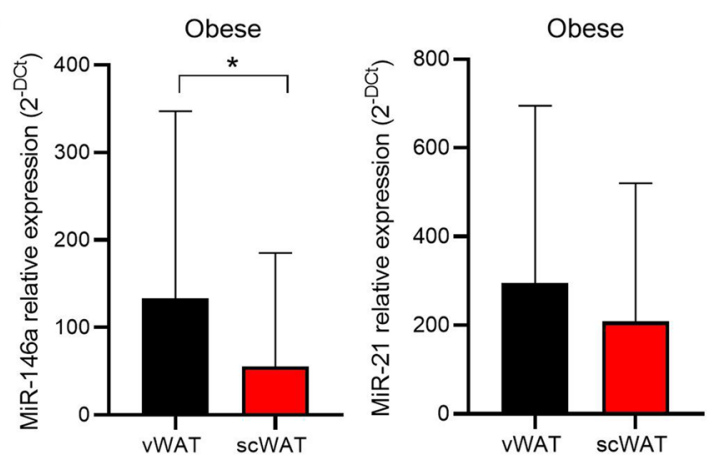

B

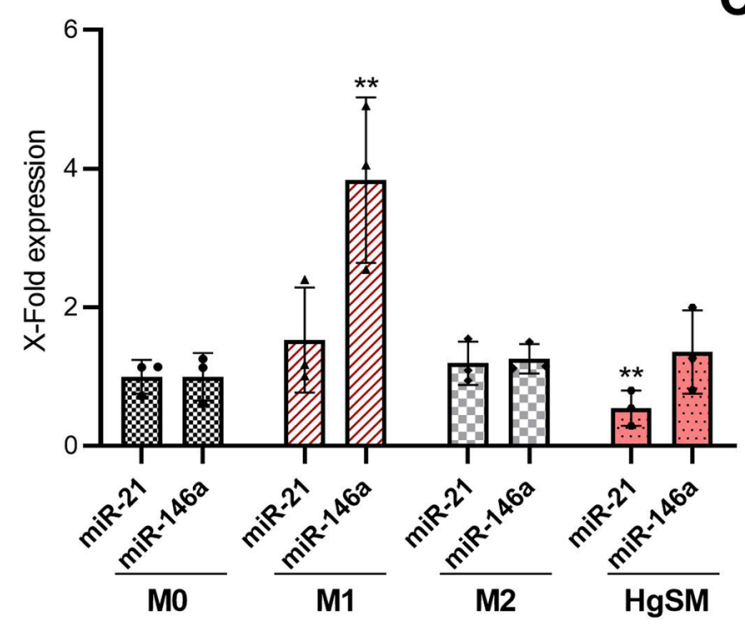

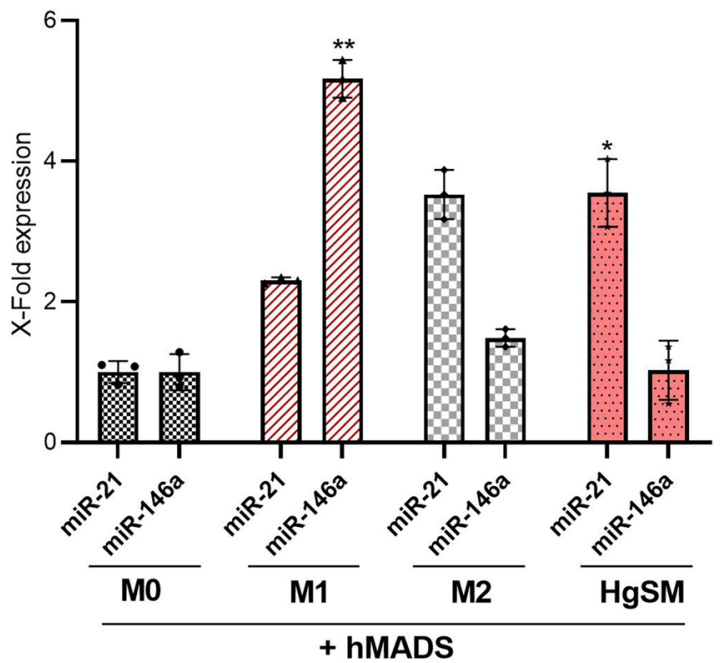

D

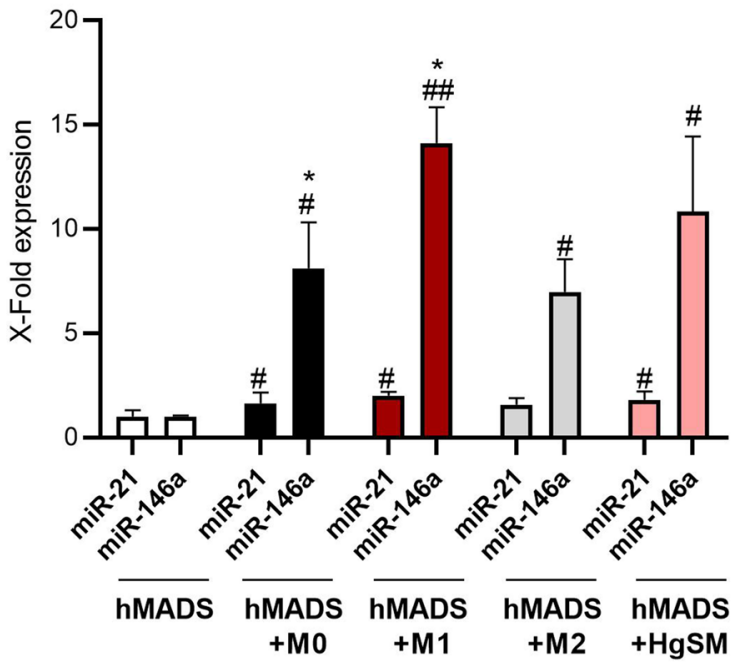

as immunoparalysis, as it occurs in patients affected by sepsis and septic shock $[51,52]$.
Of note, a mixed pro- and anti-inflammatory phenotype was previously observed in both macrophages 
4Figure 7 Analysis of miRNA expression in adipose tissue, THP-1-induced macrophages, and hMADS adipocytes in cocultured condition. A miRNA expression analysis by RT-PCR of miR-146a and miR-21 in vWAT and scWAT. B miRNA expression analysis by qRT-PCR of miR-21 and miR-146a in M0, M1, M2, and HgSM macrophages. C miRNA expression analysis by qRT-PCR of miR-21 and miR-146a in M0, M1, M2, and $\mathrm{HgSM}$ macrophages co-cultured with hMADS adypocytes. D miRNA expression analysis by qRT-PCR of miR21 and miR-146a in hMADS adipocytes co-cultured with M0, M1, M2, and HgSM macrophages. Data are expressed as mean $\pm \mathrm{SD},{ }^{*} p \leq 0.05 ; * * p \leq 0.01 ; * * * p \leq 0.001 ;{ }^{*} p \leq 0.05 ;{ }^{\# \#} p \leq 0.01$; ${ }^{\# \#} p \leq 0.001$. *vs M0; \#vs hMADS

with senescent features [10], but also in senescent endothelial cells [53, 54]. Worth mentioning, albeit the SASP is usually accompanied by a set of shared core proteins, the resulting secretory phenotype of $\mathrm{SCs}$ is highly dependent of the pro-senescence stimulus but also of the cell type involved [55].

A shift of circulating monocytes in aged people toward non-canonical pro-inflammatory phenotypes has been previously described [56, 57]. Moreover, the phenotype of aged macrophages resembles that of type 2 anti-inflammatory macrophages, partially reprogrammed toward a pro-inflammatory phenotype [58].

Because systemic inflammation with aging depends on a complex balance between inflammaging and anti-inflammaging mechanisms, the complex metabolic reshape occurring during aging (macroph-aging) may turn to be crucial to explain the onset of (adipose) tissue inflammation and to describe its systemic effects $[50,59,60]$.

During the progression of obesity, an accumulation of macrophages and other immune cells occurs in adipose tissue [61, 62], and this global reshape of tissue resident immune cells may contribute to the adipose tissue inflammatory derangement that occurs with aging, particularly in obese subjects [63]. Indeed, the interaction of $\mathrm{HgSMs}$ with adipocytes could affect their features and polarization. The co-culture of THP-1-derived macrophages with hMADS adipocytes revealed a shift toward a proinflammatory status in presence of $\mathrm{HgSMs}$. To disentangle the molecular mechanisms involved in such proinflammatory shift, we analyzed some molecules belonging to NF-kB pathway, the main proinflammatory signaling in human cells [64]. Notably, the NF-kB signaling pathway is also involved in sensitivity/resistance to insulin [65]. NF-kB activity is mediated by the activation of the IKK complex, which in turn leads to $\operatorname{IKB} \alpha$ degradation with the consequent release of the NF-kB dimers that translocate in the nucleus modulating target gene expression [29]. The modulation of the IKB- $\alpha$ protein confirmed the presence of pro-inflammatory metabolic switch in $\mathrm{HgSM}$ s when co-cultured with hMADS.

Interestingly, also hMADS adipocytes showed an important upregulation of several pro-inflammatory factors, such as IL-6, IL- $1 \beta$, TNF- $\alpha$, IL-8, and MCP1 when co-cultured with $\mathrm{HgSM}$ macrophages, corroborating the notion that macrophage secretome can foster bystander, low-grade inflammation. Consistently, in $\mathrm{HgSMs}$-treated hMADS adipocytes, the insulin signaling pathway was substantially inhibited. In insulin-treated hMADS adipocytes co-cultured with $\mathrm{HgSMs}$, we observed a significant reduction of pAKT levels, thus point at an activity of that $\mathrm{HgSMs}$-derived factors to inhibit pAKT insulin-dependent signaling [66]. This effect of HgSMs on hMADS adipocytes was accompanied by a marked reduction in IRS 1 and GLUT4 expression. TNF- $\alpha$ is known to impair insulin signaling in adipocytes by decreasing Akt levels, and levels of molecules that mediate the effects of insulin, including IRS1 and GLUT4 [67-69]. In addition, IL-6 secreted by macrophages also impairs insulin signaling in adipocytes and multiple animal models [70]. Furthermore, IL-1 $\beta$ released by macrophages mediates macrophage-induced impairment of insulin signaling pathway in human primary adipocytes [71]. Hence, a complex pro-inflammatory secretome from $\mathrm{HgSMs}$ may concur in setting off insulin resistance in hMADS. Our data showed that HgSM macrophages co-cultured with hMADS adipocytes exhibited an increased JNK phosphorylation. c-Jun NH (2)-terminal kinase (JNK) signaling pathway contributes to inflammation and to play a key role in the metabolic reshape associated with obesity [30]. In particular, JNK-deficient macrophages of mice fed with a highfat diet remained insulin-sensitive and JNK inactivation in myeloid cells led to reducing macrophage accumulation in the adipose tissue and the expression of M1 cytokines [30]. The data above support the notion that JNK signaling pathway may be involved in the induction of insulin resistance in human adipocytes exposed to HgSMs.

SIRT1 has been shown to protect against obesity; indeed, the ablation in animal model of SIRT1 in adipocyte or macrophages exacerbates obesity-induced 
metabolic dysregulation [72, 73]. Moreover, it is known that SIRT1 in adipocytes modulates expression of several adipokines including adiponectin and MCP1 [72]. In line with these findings, our data showed that $\mathrm{HgSM}$ macrophages promoted in hMADS adipocytes a reduction of SIRT1 accompanied by an increased expression of MCP1 and reduced expression of adiponectin.

Finally, we analyzed the expression levels of some inflammation-related microRNAs, named inflammamiRs [13], in HgSMs and in vWAT compared to scWAT samples of obese subjects. We observed a significantly reduction of miR-21 expression in $\mathrm{HgSMs}$ compared to M1 and M2 differentiated THP-1 cells. Interestingly, the co-culture with hMADS was associated with an increased expression of miR-21 in HgSMs. These results are in line with recent evidence suggesting that the overexpression of miR-21 significantly upregulates the pro-inflammatory cytokines, pushing the cells toward a pro-inflammatory phenotype, with partial involvement of NF-kB signal pathway [74].

In tissue samples of obese patients, we observed a significant increased level of miR-146a in vWAT compared to scWAT, suggesting that miR-146a overexpression may be a mechanism to restrain inflammation [75]. This mechanism seems not efficient in $\mathrm{HgSMs}$ and hMADS adipocytes, suggesting a derailment toward an inflammatory status. Speculatively, other (immune) cell types different from macrophages and adipocytes could be involved in miR-146a hyper-expression in vWAT.

\section{Conclusion}

Obesity is a major trigger of chronic inflammation, promoting the development of diabetes. However, no druggable target halting this pathological process has emerged so far [76]. Among others, SCs have been suggested as potential mediators of obesity-induced inflammation. Here we demonstrate for the first time that a sizeable amount of SCs in the adipose tissue of obese human subjects are macrophages (SA- $\beta-$ Gal and CD68 + cells) and that the number of SCs resident in the vWAT correlate with IR. These results, coupled with the observed ability of in vitro reproduced senescent macrophages to spread inflammation and promote IR in hMADS, corroborate a framework where SCs, and in particular senescent macrophages, might contribute to adipose tissue inflammation and diabetes development. Given that SCs are fast becoming druggable targets [77] and considering that SC removal has already been shown to improve glucose tolerance and enhance insulin sensitivity in obese mice [11], the results presented here encourage the design of further studies [40] testing the effect of SCs removal, or other safe strategies reducing the deleterious effect of SCs, on diabetes-related endpoints in obese subjects.

Author contribution G. M. and J. P.: writing-original draft; project administration; and conceptualization; E. D. M.: methodology; J. S.: writing-review and editing; and visualization; F. P.: writing-review and editing; M. S.: methodology; G. S., C. D., G. L., and M. G.: resources; A. G.: writingreview and editing, and supervision; M. B.: writing-review and editing; and conceptualization; F. O.: writing-review and editing; conceptualization; and funding acquisition.

Funding Open access funding provided by Università Politecnica delle Marche within the CRUI-CARE Agreement. This work was supported by grants from Università Politecnica delle Marche awarded to Fabiola Olivieri.

Data availability All data generated or analyzed during this study are included in this published article.

\section{Declarations}

Competing interests The authors declare no competing interests.

Open Access This article is licensed under a Creative Commons Attribution 4.0 International License, which permits use, sharing, adaptation, distribution and reproduction in any medium or format, as long as you give appropriate credit to the original author(s) and the source, provide a link to the Creative Commons licence, and indicate if changes were made. The images or other third party material in this article are included in the article's Creative Commons licence, unless indicated otherwise in a credit line to the material. If material is not included in the article's Creative Commons licence and your intended use is not permitted by statutory regulation or exceeds the permitted use, you will need to obtain permission directly from the copyright holder. To view a copy of this licence, visit http://creativecommons.org/licenses/by/4.0/.

\section{References}

1. Franceschi, C., et al., Inflamm-aging. An evolutionary perspective on immunosenescence. Ann N Y Acad Sci. 2000;908:244-254. 
2. Fulop $\mathrm{T}$, et al. The integration of inflammaging in agerelated diseases. Semin Immunol. 2018;40:17-35.

3. Reilly SM, Saltiel AR. Adapting to obesity with adipose tissue inflammation. Nat Rev Endocrinol. 2017;13(11):633-43.

4. Wellen KE, Hotamisligil GS. Inflammation, stress, and diabetes. J Clin Invest. 2005;115(5):1111-9.

5. Frasca D, Blomberg BB, Paganelli R. Aging, obesity, and inflammatory age-related diseases. Front Immunol. 2017;8:1745.

6. Haase, C.L., et al., Body mass index and risk of obesityrelated conditions in a cohort of 2.9 million people: evidence from a UK primary care database. Obes Sci Pract. 2021;7(2):137-147.

7. Baker DJ, et al. Naturally occurring p16(Ink4a)positive cells shorten healthy lifespan. Nature. 2016;530(7589):184-9.

8. Coppe JP, et al. Senescence-associated secretory phenotypes reveal cell-nonautonomous functions of oncogenic RAS and the p53 tumor suppressor. PLoS Biol. 2008;6(12):2853-68.

9. Palmer AK, et al. Cellular senescence in type 2 diabetes: a therapeutic opportunity. Diabetes. 2015;64(7):2289-98.

10. Prattichizzo F, et al. Short-term sustained hyperglycaemia fosters an archetypal senescence-associated secretory phenotype in endothelial cells and macrophages. Redox Biol. 2018;15:170-81.

11. Palmer, A.K., et al., Targeting senescent cells alleviates obesity-induced metabolic dysfunction. Aging Cell. 2019;18(3):e12950.

12. Quinn SR, O'Neill LA. A trio of microRNAs that control Toll-like receptor signalling. Int Immunol. 2011;23(7):421-5.

13. Olivieri, F., et al., miR-21 and miR-146a: The microRNAs of inflammaging and age-related diseases. Ageing Res Rev. 2021;70:101374.

14. Olivieri F, et al. MicroRNAs linking inflamm-aging, cellular senescence and cancer. Ageing Res Rev. 2013;12(4):1056-68.

15. Kangas R, et al. Circulating miR-21, miR-146a and Fas ligand respond to postmenopausal estrogen-based hormone replacement therapy-a study with monozygotic twin pairs. Mech Ageing Dev. 2014;143-144:1-8.

16. Lhamyani $\mathrm{S}$, et al. miR-21 mimic blocks obesity in mice: a novel therapeutic option. Mol Ther Nucleic Acids. 2021;26:401-16.

17. Roos J, et al. miR-146a regulates insulin sensitivity via NPR3. Cell Mol Life Sci. 2021;78(6):2987-3003.

18. Sabbatinelli, J., et al., Decreased serum levels of the inflammaging marker miR-146a are associated with clinical non-response to tocilizumab in COVID-19 patients. Mech Ageing Dev. 2021;193:111413.

19. La Sala L, et al. Circulating microRNA-21 is an early predictor of ROS-mediated damage in subjects with high risk of developing diabetes and in drug-naive T2D. Cardiovasc Diabetol. 2019;18(1):18.

20. Smith U, et al. Cellular senescence and its role in white adipose tissue. Int J Obes (Lond). 2021;45(5):934-43.

21. Minamino $\mathrm{T}$, et al. A crucial role for adipose tissue p53 in the regulation of insulin resistance. Nat Med. 2009;15(9):1082-7.
22. Schafer MJ, et al. Exercise prevents diet-induced cellular senescence in adipose tissue. Diabetes. 2016;65(6):1606-15.

23. Rouault $\mathrm{C}$, et al. Senescence-associated beta-galactosidase in subcutaneous adipose tissue associates with altered glycaemic status and truncal fat in severe obesity. Diabetologia. 2021;64(1):240-54.

24. Ghosh AK, et al. Adipose tissue senescence and inflammation in aging is reversed by the young milieu. J Gerontol A Biol Sci Med Sci. 2019;74(11):1709-15.

25. Xu, M., et al., Targeting senescent cells enhances adipogenesis and metabolic function in old age. Elife. 2015;4:e12997.

26. Hall BM, et al. Aging of mice is associated with p16(Ink4a)and beta-galactosidase-positive macrophage accumulation that can be induced in young mice by senescent cells. Aging (Albany NY). 2016;8(7):1294-315.

27. Perugini $\mathrm{J}$, et al. Biological effects of ciliary neurotrophic factor on hMADS adipocytes. Front Endocrinol (Lausanne). 2019;10:768.

28. Murano I, et al. Dead adipocytes, detected as crown-like structures, are prevalent in visceral fat depots of genetically obese mice. J Lipid Res. 2008;49(7):1562-8.

29. Giridharan S, Srinivasan M. Mechanisms of NF-kappaB p65 and strategies for therapeutic manipulation. J Inflamm Res. 2018;11:407-19.

30. Han MS, et al. JNK expression by macrophages promotes obesity-induced insulin resistance and inflammation. Science. 2013;339(6116):218-22.

31. Fuster JJ, et al. Obesity-induced changes in adipose tissue microenvironment and their impact on cardiovascular disease. Circ Res. 2016;118(11):1786-807.

32. Huang X, et al. The PI3K/AKT pathway in obesity and type 2 diabetes. Int J Biol Sci. 2018;14(11):1483-96.

33. Prattichizzo F, et al. Prevalence of residual inflammatory risk and associated clinical variables in patients with type 2 diabetes. Diabetes Obes Metab. 2020;22(9):1696-700.

34. Wu H, Ballantyne CM. Metabolic inflammation and insulin resistance in obesity. Circ Res. 2020;126(11):1549-64.

35. Coppe JP, et al. The senescence-associated secretory phenotype: the dark side of tumor suppression. Annu Rev Pathol. 2010;5:99-118.

36. Lumeng $\mathrm{CN}$, et al. Aging is associated with an increase in $\mathrm{T}$ cells and inflammatory macrophages in visceral adipose tissue. J Immunol. 2011;187(12):6208-16.

37. Espinosa De Ycaza, A.E., et al., Senescent cells in human adipose tissue: A cross-sectional study. Obesity (Silver Spring). 2021;29(8):1320-1327.

38. Conley SM, et al. Human obesity induces dysfunction and early senescence in adipose tissue-derived mesenchymal stromal/stem cells. Front Cell Dev Biol. 2020;8:197.

39. Justice JN, et al. Cellular senescence biomarker p16INK4a+ cell burden in thigh adipose is associated with poor physical function in older women. J Gerontol A Biol Sci Med Sci. 2018;73(7):939-45.

40. Hickson LJ, et al. Senolytics decrease senescent cells in humans: preliminary report from a clinical trial of Dasatinib plus Quercetin in individuals with diabetic kidney disease. EBioMedicine. 2019;47:446-56.

41. Prattichizzo F, et al. "Inflammaging" as a druggable target: a senescence-associated secretory phenotype-centered view of type 2 diabetes. Oxid Med Cell Longev. 2016;2016:1810327. 
42. Xu M, et al. JAK inhibition alleviates the cellular senescence-associated secretory phenotype and frailty in old age. Proc Natl Acad Sci U S A. 2015;112(46):E6301-10.

43. Li Q, et al. Obesity and hyperinsulinemia drive adipocytes to activate a cell cycle program and senesce. Nat Med. 2021;27(11):1941-53.

44. Villaret A, et al. Adipose tissue endothelial cells from obese human subjects: differences among depots in angiogenic, metabolic, and inflammatory gene expression and cellular senescence. Diabetes. 2010;59(11):2755-63.

45. Frasca D, Blomberg BB. Adipose tissue, immune aging, and cellular senescence. Semin Immunopathol. 2020;42(5):573-87.

46. Abbasalizad-Farhangi M. Central obesity accelerates leukocyte telomere length (LTL) shortening in apparently healthy adults: a systematic review and meta-analysis. Crit Rev Food Sci Nutr. 2021;1:1-9.

47. Olivieri F, et al. MiR-21-5p and miR-126a-3p levels in plasma and circulating angiogenic cells: relationship with type 2 diabetes complications. Oncotarget. 2015;6(34):35372-82.

48. Hall BM, et al. p16(Ink4a) and senescence-associated beta-galactosidase can be induced in macrophages as part of a reversible response to physiological stimuli. Aging (Albany NY). 2017;9(8):1867-84.

49. Liu JY, et al. Cells exhibiting strong p16 (INK4a) promoter activation in vivo display features of senescence. Proc Natl Acad Sci U S A. 2019;116(7):2603-11.

50. Prattichizzo F, et al. Senescence associated macrophages and "macroph-aging": are they pieces of the same puzzle? Aging (Albany NY). 2016;8(12):3159-60.

51. Calfee, C.S., Analysis of immunoparalysis. Sci Transl Med. 2012;4(160):160ec208. p. 160ec208.

52. Moganti K, et al. Hyperglycemia induces mixed M1/M2 cytokine profile in primary human monocyte-derived macrophages. Immunobiology. 2017;222(10):952-9.

53. Coleman PR, et al. Stress-induced premature senescence mediated by a novel gene, SENEX, results in an anti-inflammatory phenotype in endothelial cells. Blood. 2010;116(19):4016-24.

54. Coleman PR, et al. Age-associated stresses induce an antiinflammatory senescent phenotype in endothelial cells. Aging (Albany NY). 2013;5(12):913-24.

55. Hernandez-Segura, A., et al., Unmasking transcriptional heterogeneity in senescent cells. Curr Biol. 2017;27(17):2652-2660 e4.

56. Russo L, Lumeng CN. Properties and functions of adipose tissue macrophages in obesity. Immunology. 2018;155(4):407-17.

57. Costantini A, et al. Age-related M1/M2 phenotype changes in circulating monocytes from healthy/unhealthy individuals. Aging (Albany NY). 2018;10(6):1268-80.

58. van Beek AA, et al. Metabolic alterations in aging macrophages: ingredients for inflammaging? Trends Immunol. 2019;40(2):113-27.

59. Sebastian $\mathrm{C}$, et al. MacrophAging: a cellular and molecular review. Immunobiology. 2005;210(2-4):121-6.

60. Plowden $\mathbf{J}$, et al. Innate immunity in aging: impact on macrophage function. Aging Cell. 2004;3(4):161-7.
61. Lumeng CN, Bodzin JL, Saltiel AR. Obesity induces a phenotypic switch in adipose tissue macrophage polarization. J Clin Invest. 2007;117(1):175-84.

62. Olefsky JM, Glass CK. Macrophages, inflammation, and insulin resistance. Annu Rev Physiol. 2010;72:219-46.

63. Huh JY, et al. Crosstalk between adipocytes and immune cells in adipose tissue inflammation and metabolic dysregulation in obesity. Mol Cells. 2014;37(5):365-71.

64. Liu T, et al. NF-kappaB signaling in inflammation. Signal Transduct Target Ther. 2017;2:17023.

65. Baker RG, Hayden MS, Ghosh S. NF-kappaB, inflammation, and metabolic disease. Cell Metab. 2011;13(1):11-22.

66. Summers SA, Whiteman EL, Birnbaum MJ. Insulin signaling in the adipocyte. Int $\mathbf{J}$ Obes Relat Metab Disord. 2000;24(Suppl 4):S67-70.

67. Medina EA, et al. Tumor necrosis factor- $\{$ alpha $\}$ decreases Akt protein levels in 3T3-L1 adipocytes via the caspase-dependent ubiquitination of Akt. Endocrinology. 2005;146(6):2726-35.

68. Ruan H, Lodish HF. Regulation of insulin sensitivity by adipose tissue-derived hormones and inflammatory cytokines. Curr Opin Lipidol. 2004;15(3):297-302.

69. Hotamisligil GS, Shargill NS, Spiegelman BM. Adipose expression of tumor necrosis factor-alpha: direct role in obesity-linked insulin resistance. Science. 1993;259(5091):87-91.

70. Rotter V, Nagaev I, Smith U. Interleukin-6 (IL-6) induces insulin resistance in 3T3-L1 adipocytes and is, like IL-8 and tumor necrosis factor-alpha, overexpressed in human fat cells from insulin-resistant subjects. J Biol Chem. 2003;278(46):45777-84.

71. Gao D, et al. Interleukin-1beta mediates macrophageinduced impairment of insulin signaling in human primary adipocytes. Am J Physiol Endocrinol Metab. 2014;307(3):E289-304.

72. Hui X, et al. Adipocyte SIRT1 controls systemic insulin sensitivity by modulating macrophages in adipose tissue. EMBO Rep. 2017;18(4):645-57.

73. Mayoral R, et al. Adipocyte SIRT1 knockout promotes PPARgamma activity, adipogenesis and insulin sensitivity in chronic-HFD and obesity. Mol Metab. 2015;4(5):378-91.

74. Madhyastha R, et al. MicroRNA 21 Elicits a pro-inflammatory response in macrophages, with exosomes functioning as delivery vehicles. Inflammation. 2021;44(4):1274-87.

75. Runtsch, M.C., et al., Anti-inflammatory microRNA-146a protects mice from diet-induced metabolic disease. PLoS Genet. 2019;15(2):e1007970.

76. Pollack RM, et al. Anti-inflammatory agents in the treatment of diabetes and its vascular complications. Diabetes Care. 2016;39(Suppl 2):S244-52.

77. Gurau F, et al. Anti-senescence compounds: a potential nutraceutical approach to healthy aging. Ageing Res Rev. 2018;46:14-31.

Publisher's note Springer Nature remains neutral with regard to jurisdictional claims in published maps and institutional affiliations. 